ESAIM: M2AN 49 (2015) 529-550

DOI: $10.1051 / \mathrm{m} 2 \mathrm{an} / 2014042$
ESAIM: Mathematical Modelling and Numerical Analysis

www.esaim-m2an.org

\title{
ERROR ESTIMATE FOR A FINITE VOLUME SCHEME IN A GEOMETRICAL MULTI-SCALE DOMAIN
}

\author{
MARIE-Claude Viallon ${ }^{1}$
}

\begin{abstract}
We study a finite volume scheme, introduced in a previous paper [G.P. Panasenko and M.-C. Viallon, Math. Meth. Appl. Sci. 36 (2013) 1892-1917], to solve an elliptic linear partial differential equation in a rod structure. The rod-structure is two-dimensional (2D) and consists of a central node and several outgoing branches. The branches are assumed to be one-dimensional (1D). So the domain is partially $1 \mathrm{D}$, and partially 2D. We call such a structure a geometrical multi-scale domain. We establish a discrete Poincaré inequality in terms of a specific $H^{1}$ norm defined on this geometrical multi-scale 1D-2D domain, that is valid for functions that satisfy a Dirichlet condition on the boundary of the $1 \mathrm{D}$ part of the domain and a Neumann condition on the boundary of the $2 \mathrm{D}$ part of the domain. We derive an $L^{2}$ error estimate between the solution of the equation and its numerical finite volume approximation.
\end{abstract}

Mathematics Subject Classification. 35J25, 74S10, 65N12, 65N15, 65N08.

Received January 28, 2014. Revised July 20, 2014.

Published online 17 March 2015.

\section{INTRODUCTION}

This paper is concerned with a finite volume scheme for a geometrical multi-scale domain. We obtain a specific discrete Poincaré inequality for that type of structure. This inequality is then used to improve from $O(\sqrt{h})$ to $O(h)$ a first error estimate obtained in [36] for a simple model problem.

The plan is as follows. In Section 1.1, we present the background in which the dimensionally-heterogeneous modelling takes place. Then, in Section 1.2, we describe in detail the geometrical multi-scale 1D-2D domain on which our problem is set (see Fig. 1). The parameter $\varepsilon$ is related to the width of the branches. In the following section we define our model problem (1.1). Then in Section 1.4, the main result of the paper, that is error estimates in specific $H^{1}$ and $L^{2}$ norms using the finite volume scheme introduced in [36], is discussed. These results improve a previous estimate that is reminded in Section 1.5. Section 1.6 is devoted to the review of different ways to state interface conditions between domains of different dimensions, Section 1.7 to the review of discrete Poincaré inequalities, especially for functions that vanish only on a part of the boundary. In Section 1.8, from a numerical standpoint, we compare the dimension reduction of the domain to the use of non-matching grids, taking a row of big cells. Then some remarks on the domain decomposition approach end Section 1.

Keywords and phrases. Finite volume scheme, elliptic problem, discrete Poincaré inequality, error estimate, multi-scale domain.

1 Université de Lyon, UMR CNRS 5208, Université Jean Monnet, Institut Camille Jordan, Faculté des Sciences et Techniques,

23 rue Docteur Paul Michelon, 42023 Saint-Etienne cedex 2, France. jf.us.gerbeau@gmail.com 
In Section 2, we present our hybrid scheme to solve (1.1). We prove that the scheme gives a unique discrete solution. In Section 3, following [19], we define a $H^{1}$ discrete norm and we establish a discrete Poincaré inequality. Last in Section 4, we derive the error estimates previously announced in Section 1.4.

\subsection{The dimensionally-heterogeneous modelling}

This paper deals with the resolution of a model problem set in a finite rod structure. A finite rod structure is a connected finite union of cylinders (rectangles in the two-dimensional case). Arterial trees in the cardiovascular system, systems of pipes in industrial installations, or canal systems, are classical examples of rod structures. Since the direct numerical solution of partial differential equations in such domains implies high computational costs, we use an alternative approach: we reduce the costs by considering the rods as one-dimensional domains, yet keeping the junctions as two or three-dimensional domains. This therefore leads to work in a geometrical multi-scale domain (a single numerical model with different space scales). The usefulness of the coupling of models of different dimensions has been shown for instance in $[6,9,22,23,41]$. The main application area is computational hemodynamics. Dimensionally-heterogeneous modelling has been applied to describe the relationship between the local blood flow patterns and the global hemodynamic environments for instance in [9,23,41]. In [38], the authors present a model where a 1D description of the circle of Willis (cerebral vasculature) is coupled to a fully three-dimensional (3D) model of a carotid artery. The 3D model is well suited for investigating the effects of the geometry on the blood flow on a space scale of a few centimeters. By exploiting the cylindrical geometry of vessels, it is possible to resort to $1 \mathrm{D}$ models, by reducing the space dependence to the vessel's axial coordinate only. The 1D models are convenient when the interest is in obtaining the pressure dynamics in a large part of the vascular tree at a reasonable computational cost. This geometrical multi-scale approach has been proposed in [22], some difficulties arising from the coupling have been discussed in [24]. In [38], the authors point out that their approach can be extended to hydraulic networks featuring pipes. Rather, in [33], the authors deal with dimensionally-heterogeneous hydraulic networks. Yet, the coupling of partial differential equations is of increasing importance for industrial applications, and namely the geometrical multi-scale problems. Such a coupling arises for instance in the simulation of the flow in the primary coolant circuit of a pressurized water reactor in a nuclear power plant: one may use a $1 \mathrm{D}$ code to deal with the pipes and a $3 \mathrm{D}$ code to model the reactors. In $[2,10]$, the authors focused on a coupling condition at the interface among domains that all have the same geometrical dimension. Though, the coupling of 1D and 2D CFD codes is discussed in [28], where the coupling of the 1D isentropic Euler system to the 2D one is considered: an associated 1D Riemann problem is solved at the interface between the two systems. This work has been extended recently in [16] to the coupling of a density-based 3D Euler code to a 1D version of the code (for instance, an application is the simulation of diesel injectors).

However, in this paper, we solve a model problem in a simple 2D rod structure. We do not consider a realistic model such as described above. It is a first step. Extensions to more realistic problems are possible.

\subsection{Description of the geometrical multi-scale 1D-2D domain}

Before introducing the 1D-2D domain on which our model problem is set, let us look at the following example of finite rod structures. It consists of one node and $n$ branches. This construction is done in [36] and is discussed below.

Let $e_{j}=\left[O, O_{j}\right], j=1, \ldots, n$, be $n$ closed segments in $\mathbb{R}^{2}$, having a common end point denoted by $O$, with length $l_{j}=O O_{j}, j=1, \ldots, n$.

Let $(x, y)$ denote the coordinates in the canonical basis of $\mathbb{R}^{2}$, and $\left(x^{e_{j}}, y^{e_{j}}\right)$ denote the local coordinates associated with the segment $e_{j}, j=1, \ldots, n$. This local system is orthonormal and such that $x^{e_{j}}$ is the coordinate in the direction $e_{j}$.

Let $\varepsilon>0$. Let $\theta_{1}, \ldots, \theta_{n}$ be positive numbers independent of $\varepsilon$.

Let $B_{j}^{\varepsilon}=\left\{(x, y) \mid x^{e_{j}} \in\left(0, l_{j}\right), \quad y^{e_{j}} \in\left(-\frac{\varepsilon \theta_{j}}{2}, \frac{\varepsilon \theta_{j}}{2}\right)\right\}$, and $\hat{\beta}_{j}^{\varepsilon}=\left\{(x, y) \mid x^{e_{j}}=l_{j}, \quad y^{e_{j}} \in\left(-\frac{\varepsilon \theta_{j}}{2}, \frac{\varepsilon \theta_{j}}{2}\right)\right\}$. 

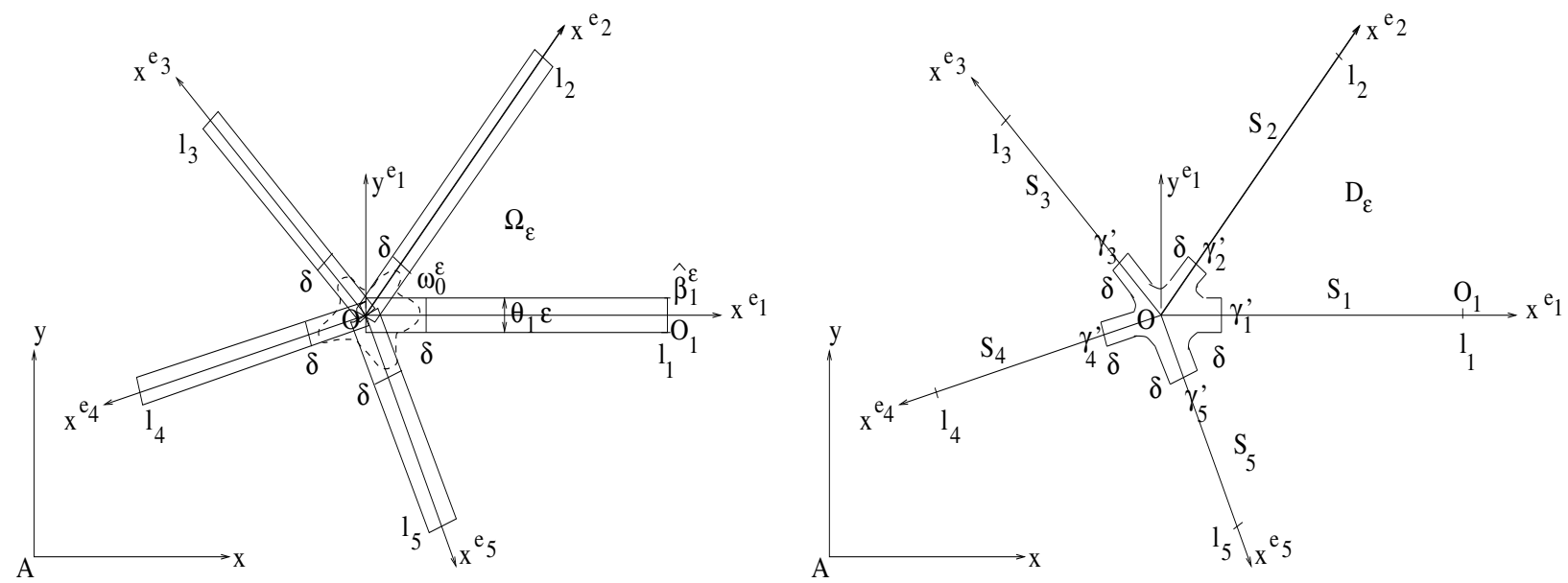

FiguRE 1. (a) The initial domain $\Omega_{\varepsilon}$ and (b) the geometrical multi-scale domain $D_{\varepsilon}$.

Let $\omega_{0}$ be a bounded domain in $\mathbb{R}^{2}$ with smooth boundary containing $O$ (see [36]). Let $\omega_{0}^{\varepsilon}=\left\{(x, y) \mid \frac{(x, y)-O}{\varepsilon} \in\right.$ $\left.\omega_{0}\right\}$. We assume that $B_{j}^{\varepsilon} \backslash \omega_{0}^{\varepsilon} \cap B_{i}^{\varepsilon} \backslash \omega_{0}^{\varepsilon}=\emptyset, i \neq j$. The domain $\omega_{0}^{\varepsilon}$ (see the dotted line in Fig. 1a) is added in order to smooth the boundary of the final structure by removing the corners.

Let $\Omega_{\varepsilon}=\cup_{j=1}^{n} B_{j}^{\varepsilon} \cup \omega_{0}^{\varepsilon}$. The domain $\Omega_{\varepsilon}$ is thus the $1 / \varepsilon-$ homothetic contraction of a fixed domain $\Omega$, as depicted in Figure 1a with $n=5$. The thickness of the branches is the ratio of the diameter to the height, and is proportional to $\varepsilon$.

Now, let us describe the 1D-2D domain under consideration. Let $\delta>0$, such that $\delta<\min \left\{l_{j}, j=1, \ldots, n\right\}$ and such that $\omega_{0}^{\varepsilon}$ is in the ball of center $O$ and radius $\delta$.

Denote ${B^{\prime}}_{j}^{\varepsilon}=B_{j}^{\varepsilon} \cap\left\{(x, y) \quad \mid x^{e_{j}} \in(0, \delta)\right\}, j=1, \ldots, n$. Denote $\Omega_{\varepsilon}^{\prime}=\cup_{j=1}^{n} B_{j}{ }_{j} \cup \omega_{0}^{\varepsilon}$. So $\Omega_{\varepsilon}^{\prime}$ is a truncated part of the initial domain $\Omega_{\varepsilon}$.

Let $S_{j}=\left\{(x, y) \mid y^{e_{j}}=0, x^{e_{j}} \in\left(\delta, l_{j}\right)\right\}, j=1, \ldots, n$, be segments such that $S_{j} \subset e_{j}$.

We denote $\gamma_{j}^{\prime}=\left\{(x, y) \mid x^{e_{j}}=\delta, \quad y^{e_{j}} \in\left(-\frac{\varepsilon \theta_{j}}{2}, \frac{\varepsilon \theta_{j}}{2}\right)\right\}, j=1, \ldots, n$, the interfaces between $\Omega_{\varepsilon}^{\prime}$ and $\Omega_{\varepsilon} \backslash \Omega_{\varepsilon}^{\prime}$ (for the sake of simplicity, we do not make the dependence on $\varepsilon$ of $\gamma_{j}^{\prime}$ ).

Let us define $D_{\varepsilon}=\Omega_{\varepsilon}^{\prime} \cup\left(\cup_{j=1}^{n} S_{j}\right)$. The set $D_{\varepsilon}$ is what we call a geometrical multi-scale domain. We assume that $\omega_{0}^{\varepsilon} \backslash \cup_{j=1}^{n} B_{j}^{\varepsilon}$ is not too large. More precisely, we assume that $m\left(\Omega_{\varepsilon}^{\prime}\right)$ is of the same magnitude as $m\left(\cup_{j=1}^{n} B_{j}^{\varepsilon}\right)$, so as to have $m\left(\Omega_{\varepsilon}^{\prime}\right)=O(\varepsilon \delta)$, where $m$ is the 2 D Lebesgue measure.

In this paper, we consider both the case of a geometrical multi-scale domain where $\varepsilon$ and $\delta$ are fixed, and the case where $\varepsilon$ tends to zero and $\delta$ depends on $\varepsilon$. The two studies are made at the same time, and Theorems 4.1 and 4.4 are stated in Section 4 related to each case.

\subsection{The model problem}

The boundary value problem in the domain $D_{\varepsilon}$, that we consider in this paper, is the following:

$$
\left\{\begin{array}{l}
v_{j}^{\prime \prime}\left(x^{e_{j}}\right)=f_{j}\left(x^{e_{j}}\right), x^{e_{j}} \in\left(\delta, l_{j}\right), j=1, \ldots, n \text { (a) } \\
v_{j}\left(l_{j}\right)=0, j=1, \ldots, n \\
\triangle u(x, y)=0,(x, y) \in \Omega_{\varepsilon}^{\prime} \\
\frac{\partial u}{\partial n}(x, y)=0,(x, y) \in \partial \Omega_{\varepsilon}^{\prime} \backslash\left(\cup_{j=1}^{n} \gamma_{j}^{\prime}\right) \\
u(x, y)=v_{j}(\delta),(x, y) \in \gamma_{j}^{\prime}, j=1, \ldots, n \\
v_{j}^{\prime}(\delta)=\frac{1}{\theta_{j} \varepsilon} \int_{\gamma_{j}^{\prime}} \frac{\partial u}{\partial n} \mathrm{~d} \gamma, j=1, \ldots, n .
\end{array}\right.
$$


We assume that the functions $f_{j}$ are independent of $\varepsilon$ and vanish in some neighborhood of $O_{j}, j=1, \ldots, n$. For the sake of simplicity, as in [36], the right-hand side is taken equal to zero in $\Omega_{\varepsilon}^{\prime}$, but this condition could be relaxed. However, it is well known that the error estimates for the convergence rate of the numerical methods require some regularity of the exact solution. So we assume that the right-hand side is such that $u \in C^{2}\left(\overline{\Omega_{\varepsilon}^{\prime}}\right)$ and $v_{j} \in C^{2}\left(\left[\delta, l_{j}\right]\right), j=1, \ldots, n$.

More precisely, we define a global solution $u^{d}$ of (1.1) by letting

$$
u^{d}(x, y)=\left\{\begin{array}{l}
u(x, y) \text { if }(x, y) \in \Omega_{\varepsilon}^{\prime} \\
v_{j}\left(x^{e_{j}}\right) \text { if }(x, y) \in B_{j}^{\varepsilon}, x^{e_{j}} \in\left(\delta, l_{j}\right), j=1, \ldots, n .
\end{array}\right.
$$

The solution $u^{d}$ is defined in $\Omega_{\varepsilon}$ but $u^{d}(x, y)$ does not depend on $y^{e_{j}}$ when $(x, y) \in B_{j}^{\varepsilon} \backslash{B^{\prime}}_{j}^{\varepsilon}$. Defining the solution on $\Omega_{\varepsilon}$ will allow us to use a standard $L^{2}$ norm in a $2 \mathrm{D}$ domain to write the error estimate of Theorem 4.1.

Problem (1.1) has been introduced in [36] in the framework of the method of asymptotic partial domain decomposition (MAPDD) (see [35]). The following lemma has been proved in [37] (see estimate (1.6)) and [36].

Lemma 1.1. For any $J>0$, there is $M$, independent of $\varepsilon$, such that if $\delta=M \varepsilon|\ln \varepsilon|$, then

$$
\left\|u_{\varepsilon}-u^{d}\right\|_{H^{1}\left(\Omega_{\varepsilon}\right)}=O\left(\varepsilon^{J}\right),
$$

where $u_{\varepsilon}$ is the solution of the following elliptic linear model equation

$$
\left\{\begin{array}{l}
\triangle u_{\varepsilon}=f, \quad \text { in } \quad \Omega_{\varepsilon} \\
u_{\varepsilon}=0, \quad \text { on } \quad \hat{\beta}_{j}^{\varepsilon}, j=1, \ldots, n \\
\frac{\partial u_{\varepsilon}}{\partial n}=0, \quad \text { on } \quad \partial \Omega_{\varepsilon} \backslash\left(\cup_{j=1}^{n} \hat{\beta}_{j}^{\varepsilon}\right)
\end{array}\right.
$$

where $f$ is a smooth function defined in $\Omega_{\varepsilon}$ such that $f(x, y)=f_{j}\left(x^{e_{j}}\right)$, if $(x, y) \in B_{j}^{\varepsilon} \backslash{B^{\prime}}_{j}^{\varepsilon}, j=1, \ldots, n$, and $f(x, y)=0$ if $(x, y) \in \Omega_{\varepsilon}^{\prime}$.

There exists a function $u_{\varepsilon} \in C^{2}\left(\overline{\Omega_{\varepsilon}}\right)$ solution of (1.3), if $f$ is sufficiently smooth [27]. It is proved in [36] that the following estimates hold.

Lemma 1.2. If $\delta$ is of order $\varepsilon \ln \varepsilon$ then

$$
\left\|v_{j}^{\prime}\right\|_{\infty}=O(1) \quad \text { and }\left\|v_{j}^{\prime \prime}\right\|_{\infty}=O(1), j=1, \ldots, n, \quad\|\nabla u\|_{\infty}=O(1), \quad\left\|\nabla^{2} u\right\|_{\infty}=O\left(\frac{1}{\varepsilon}\right) .
$$

These bounds will be useful to prove the error estimate of Theorem 4.4.

\subsection{Comments on the numerical approximation and the error estimate}

An hybrid (in the sense that it solves a problem in a geometrical multi-scale domain) finite volume scheme is proposed in [36] to solve (1.1). To construct the scheme, the methodology which was proposed in [45] is first explained. In [45], the authors give a numerical methodology to address the solution of the 3D Navier-Stokes equations and its coupling with some $1 \mathrm{D}$ models (see $[6,7,32,33]$ also). To follow this path to solve (1.1), let us 
remark that (1.1) can be rewritten

$$
\begin{aligned}
& \left\{\begin{array}{l}
v_{j}^{\prime \prime}\left(x^{e_{j}}\right)=f_{j}\left(x^{e_{j}}\right), x^{e_{j}} \in\left(\delta, l_{j}\right), j=1, \ldots, n \\
v_{j}\left(l_{j}\right)=0, j=1, \ldots, n \\
v_{j}(\delta)=\alpha_{j},(x, y) \in \gamma_{j}^{\prime}, j=1, \ldots, n \\
v_{j}^{\prime}(\delta)=\beta_{j}
\end{array}\right. \\
& \left\{\begin{array}{l}
\triangle u(x, y)=0,(x, y) \in \Omega_{\varepsilon}^{\prime} \\
\frac{\partial u}{\partial n}(x, y)=0,(x, y) \in \partial \Omega_{\varepsilon}^{\prime} \backslash\left(\cup_{j=1}^{n} \gamma_{j}^{\prime}\right) \\
u(x, y)=\alpha_{j},(x, y) \in \gamma_{j}^{\prime}, j=1, \ldots, n \\
\frac{1}{\theta_{j} \varepsilon} \int_{\gamma_{j}^{\prime}} \frac{\partial u}{\partial n} \mathrm{~d} \gamma=\beta_{j} .
\end{array}\right.
\end{aligned}
$$

The basic idea in [45] is to consider the numerical resolution of the $2 \mathrm{D}$ problem (1.5') on one hand, and of the 1D problems (1.4') on the others hand, as black-boxes which receive the input data $\left(\alpha_{j}, j=1, \ldots, n\right)$ and give back $\left(\beta_{j}, j=1, \ldots, n\right)$ as output data. A system in the interface unknowns $\left(\alpha_{j}, \beta_{j}, j=1, \ldots, n\right)$ is obtained, which is solved by an iterative method. This technique, which is a domain decomposition approach, will not be dealt with here. Instead, in the present paper, a direct method is used, and (1.4') and (1.5') are not understood as black-boxes but related by (1.1c) (reminded below for easy reference and guidance):

$$
v_{j}^{\prime}(\delta)=\frac{1}{\theta_{j} \varepsilon} \int_{\gamma_{j}^{\prime}} \frac{\partial u}{\partial n} \mathrm{~d} \gamma, j=1, \ldots, n .
$$

Here, $\beta_{j}, j=1, \ldots, n$, are no longer unknowns and only the interface unknowns $\alpha_{j}, j=1, \ldots, n$, are kept. We use finite volume schemes to approach $\left(1.4^{\prime}\right),\left(1.5^{\prime}\right)$, and (1.6), where (1.4') (resp. (1.5')) is the system (1.4) (resp. (1.5)) with its last equation removed. The unknowns corresponding with $\alpha_{j}, j=1, \ldots, n$, are $v_{j, 0}, j=$ $1, \ldots, n$, in the resulting scheme that is recalled in (2.2) in Section 2.2.

The aim of the present paper is to reconsider this scheme to solve (1.1), and in particular to improve the order of convergence obtained in [36]. In [36], we get an error estimate of order $\sqrt{h}$, where $h$ is the size of the mesh. In Theorem 4.1 below we get a better estimate $O(h)$. This is one of the main results of the paper. Here $\varepsilon$ and $\delta$ are fixed given parameters and we don't have to express the bound with respect to these parameters.

However, in addition, (1.4'), (1.5'), (1.6) may also be used to solve (1.3). In view of Lemma 1.1, (1.1) is a reasonable approximation for (1.3) if $\varepsilon$ is small and $\delta$ of order $\varepsilon \ln \varepsilon$. So, a numerical approximation of the solution of (1.1) is also a numerical approximation of the solution of (1.3). In Section 4, an error estimate between the solution of (1.3) and its numerical approximation is obtained in Theorem 4.4 in conjunction with Theorem 4.1. Since both $h$ and $\varepsilon$ tend to zero in this case, the error estimate is also expressed in terms of $\varepsilon$. Note that a finite element implementation of (1.3) is studied in [21] with $n=1$, and an error estimate is obtained.

We obtain a better error estimate than in [36] because (1.1) is really considered as a geometrical multi-scale problem. We define discrete $L^{2}$ and $H^{1}$ norms for functions on $D_{\varepsilon}$. A $H^{1}$ discrete norm has been introduced in [42], in the case of a structure with a single branch. Here, we propose a generalization to structures with $n$ branches. It involves the convex combination of the values of the functions on both sides of each interface $\gamma_{j}^{\prime}, j=1, \ldots, n$. To the best of our knowledge, there is no error estimate in the literature when using a geometrical multi-scale finite volume scheme. Moreover, the problem (1.1) is such that Neumann boundary conditions are imposed on the 2D part of the domain, and Dirichlet boundary conditions are imposed on the boundary of the 1D part of the domain. As no classical Poincaré inequality is directly applicable on an issue of this nature, it has been necessary to establish a discrete Poincaré inequality in $D_{\varepsilon}$. 


\subsection{About the estimate in $[36]$}

We recall here how the estimate $O(\sqrt{h})$ is obtained in [36]. Let $\bar{v}_{j}, \widetilde{v}_{j}, \widetilde{u}_{j}, j=1, \ldots, n$, be the solutions of the following independent sub-problems, some of them being 1D, and the others being $2 \mathrm{D}$

$$
\left\{\begin{array} { l } 
{ \overline { v } _ { j } ^ { \prime \prime } = f _ { j } , \text { on } S _ { j } } \\
{ \overline { v } _ { j } ( \delta ) = 0 }
\end{array} \quad \left\{\begin{array} { l } 
{ \widetilde { v } _ { j } ^ { \prime \prime } = 0 , \text { on } S _ { j } } \\
{ \widetilde { v } _ { j } ( \delta ) = 1 }
\end{array} \quad \left\{\begin{array}{l}
\triangle \widetilde{u}_{j}=0, \text { on } \Omega_{\varepsilon}^{\prime} \\
\left.\widetilde{u}_{j}\right|_{\gamma_{j}^{\prime}}=1, \\
\left.\widetilde{u}_{j}\right|_{\gamma_{k}^{\prime}}=0, \text { if } k \neq j, k=1, \ldots, n .
\end{array}\right.\right.\right.
$$

The solution of (1.1) can then be written

$$
\left\{\begin{array}{l}
v_{j}=\bar{v}_{j}+\alpha_{j} \widetilde{v}_{j}, j=1, \ldots, n \\
u=\sum_{j=1}^{n} \alpha_{j} \widetilde{u}_{j}
\end{array}\right.
$$

The auxiliary variables $\alpha_{j}, j=1, \ldots, n$, are then defined by

$$
\frac{1}{\theta_{j} \varepsilon} \sum_{k=1}^{n} \alpha_{k} \int_{\gamma_{j}^{\prime}} \frac{\partial \widetilde{u}_{k}}{\partial n} \mathrm{~d} \gamma-\alpha_{j} \widetilde{v}_{j}^{\prime}(\delta)=\bar{v}_{j}^{\prime}(\delta), j=1, \ldots, n
$$

so that the interface conditions (1.1-c) are satisfied.

We remark that $\alpha_{j}=\left.u_{j}\right|_{\gamma_{j}^{\prime}}=v_{j}(\delta), j=1, \ldots, n$, are the values of the solution on the interfaces $\gamma_{j}^{\prime}$. Thanks to the linearity of (1.1), the problem has been completely split in [36]. The authors first derived the errors for each linear sub-problems (1.7) separately by using classical techniques for finite volume schemes, on one hand on the domains $S_{j}, j=1, \ldots, n$, and on the other hand on the domain $\Omega_{\varepsilon}^{\prime}$. They then deduced the error on the reconstructed solution (1.8). This is not optimal because the approximation of $\alpha_{j}, j=1, \ldots, n$, is not.

Ultimately, under the assumptions of Lemmas 1.1 and 1.2, they get an error estimate $O\left(\sqrt{\frac{h \delta}{\varepsilon}}\right)+O\left(\varepsilon^{J}\right)$ between the solution of (1.3) and its approximation. To control the errors on the interfaces, the authors need to assume that $h|\ln \varepsilon| / \varepsilon$ tends to zero when $h$ and $\varepsilon$ tend to zero, and some regularity for the mesh.

The error estimate for (1.1) is not clearly given in [36]. However, the approximation of (1.1) is a necessary step to get the one of (1.3), then it is easy to deduce from [36] an error estimate $O(\sqrt{h})$ between the solution of (1.1) and its approximation (in this case $\varepsilon$ and $\delta$ are fixed constants). The estimate is obtained under some regularity for the mesh.

So the present case is quite different since we do not need to estimate the error between $\alpha_{j}, j=1, \ldots, n$, and their approximations.

\subsection{Some remarks about the interface conditions}

In the present work, the interface conditions on $\gamma_{j}^{\prime}, j=1, \ldots, n$, in (1.1) are those induced by the MAPDD (see [35]). But the application of this method to complex problems is not yet available. Often the geometrical multi-scale modelling is achieved with the scope of delimiting the computational domain at hand in order to reduce the computational costs (see the references below). Firstly, the location of the interfaces is arbitrary. Secondly, it is difficult to determine which conditions may be assumed on the interfaces. In [30], the authors propose different artificial boundary conditions to preserve the well posedness of the Navier-Stokes problem. In [23], in the area of computational hemodynamics, the authors have treated the coupling of 3D models based on the Navier-Stokes equations with reduced 1D models, and the continuity of the cross sectional area is prescribed: numerical spurious reflections at the coupling interfaces are observed. However, the area of the vessel at both sides may differ from each other (when using elastic models), which led the authors in [41] to relax this condition, and to formulate in [6] an extended variational principle for problems where fields can become discontinuous at the coupling interfaces. The influence of the proposed interface conditions on the amplitude of the spurious 
reflections is studied in [34]. In [6], the authors point out that no reliable solutions must be expected in the regions near the coupling interfaces. In [28], the coupling of the $1 \mathrm{D}$ and the $2 \mathrm{D}$ (3D in [16]) Euler systems is done by defining admissible coupling boundary, which yields a conservative admissible interface model.

\subsection{About Poincaré inequalities}

Error estimates for numerical methods are obtained thanks to functional analysis tools, such as discrete Sobolev inequalities. Concerning the finite volume framework, and the two-dimensional case, a first discrete Poincaré inequality for piecewise constant functions has been achieved for Dirichlet boundary conditions in [13], following [29], in a polygonal convex domain. In [19], the authors generalize this inequality in a polygonal domain. Discrete Sobolev inequalities (estimating the $L^{p}$ norm) are presented in [14,17-19]. In [19, 25], the authors establish a "mean Poincaré" (Poincaré-Wirtinger) inequality (estimating the $L^{2}$ norm) for Neumann boundary conditions in a polygonal domain. A discrete "mean Poincaré" inequality (estimating the $L^{p}$ norm) is obtained in $[12,26]$ on Voronoi finite volume meshes. A Sobolev-Poincaré inequality (embedding of $W^{1, q}$ into $L^{p}$ ) was stated using a proof based on the space of functions of bounded variation in [5,20] (also in [18] for the zero boundary value case). The previous results were mostly presented in the framework of admissible meshes which satisfy the following orthogonality property: there exists a point associated with each element of the mesh such that the straight line connecting these points for two neighboring cells is orthogonal to the common side of these two cells (see the definition in [19] and (2.1) below), but more general meshes are possible (see [19]). In [43] the author presents both discrete Poincaré and "mean Poincaré" inequalities for functions defined on a mesh where the orthogonality property is not necessarily satisfied (other references in the finite element framework are given therein), as well as in [3] and [31] in the discrete duality finite volume context. Previously a discrete Poincaré inequality on non-matching grids has been established in [11]. In all the papers listed above dealing with nonconforming meshes, it is necessary to define a specific $H^{1}$ norm that is appropriate for the mesh.

In the present work, we use an admissible mesh in $\Omega_{\varepsilon}^{\prime}$, but the global mesh of $D_{\varepsilon}$ is in some ways "nonconforming". We actually define a specific $H^{1}$ norm for functions defined on $D_{\varepsilon}$ (see Sect. 1.4). In (1.1), we impose zero boundary value on the 1D part of the domain and there is a Neumann boundary condition on the 2D part of the domain, so we need to state first a discrete "mean boundary Poincaré" inequality (inequality that involves a mean value on a part of the boundary), and then to deduce a Poincaré inequality for functions with zero value on a part of the boundary. Such an inequality is obtained in [19,43], and in [5] for a convex domain. A discrete Sobolev-Poincaré inequality (estimating the $L^{p}$ norm) is established for functions with nonzero boundary values in [4]. But, these results cannot be applied to a dimensionally-heterogeneous domain. In Section 3, we follow the proof in [19], evaluating precisely the constant bounds as in [43], to get the suitable discrete Poincaré inequality that is used in Section 4 to deduce the $L^{2}$ error estimates.

\subsection{To choose a coarse grid instead of reducing the dimension?}

From the numerical standpoint, one may wonder why not to keep a fully $2 \mathrm{D}$ (or 3D in the general case) domain, and choose a coarse grid made of rectangular cells (or rectangular parallelepiped) in areas where the calculation of the solution does not require a great accuracy, rather than to reduce the dimension. This falls within the classical problems arising in domain decomposition: what are the interface conditions on the nonmatching grids? There is a wide literature on this topic, and even, more specifically, using finite volume schemes (see for instance $[1,11,40]$ ). A comparison between an hybrid scheme used on a dimensionally-heterogeneous (1D-2D) domain and the so-called TPFA scheme (defined in [19]) used on a full 2D non matching finite volume mesh, solving the Poisson equation in a rod structure with a single node and a single branch, can be found in [42]. The branch is of thickness $\varepsilon$, and meshed with a row of rectangular cells $\varepsilon$ high by $h$ wide, where $h$ is the size of the mesh of the remaining part of the domain (corresponding to the node). The a priori estimate on the error which is achieved in [42] for the TPFA scheme, following [11], depends on $\varepsilon$ for several reasons: the size of the global mesh depends on the size of the rectangles, the sum of the length of the atypical edges is equal to $\varepsilon$, and the second derivative of the solution is of the order $1 / \varepsilon$ (see Lem. 1.2). Under the assumption that $h<\varepsilon$, 
the most significant term is $O(\sqrt{\varepsilon})$, and it is impossible to get a bound with respect to $h$. Quite the contrary, the error estimate obtained in [42] for the hybrid scheme can be expressed as a function of $h$ (this result is generalized in this paper, see Thms. 4.1 and 4.4), as well as a function of $\varepsilon$. This is a main advantage of the geometrical multi-scale domain. Though, the numerical experiments in [42] show that the two schemes provide similar performances. On the other hand, a discrete Poincaré inequality for non-matching grids is obtained, for instance in [11], under the assumption of quasi-uniformness of the mesh. A discrete Poincaré inequality is used in [42] which does not require any restrictive assumption on the mesh. The proof of this inequality is not given in [42], it is a particular case of the one that is provided in the present paper (see Lem. 3.3).

\subsection{The domain decomposition approach}

In [6], the authors introduce a specialized vocabulary to name the scheme that discretizes $\left(1.4^{\prime}\right),\left(1.5^{\prime}\right),(1.6)$ : the monolithic scheme. Alternately, a decoupled numerical scheme may be devised in case of working with standalone 1D and 2D (or 3D) codes, such as black boxes. In this case we can split the computations by performing iterations between the 1D and 2D (or 3D) sub-problems. In [6], the authors called theses schemes: the segregated coupling schemes. Due to the heterogeneous feature of the geometrical multi-scale problems, the monolithic scheme gives a linear system that is ill conditioned. For this reason, many authors adopt an iterative approach by solving separately the sub-problems. For instance, the technique presented in $[32,33]$ can be understood as a domain decomposition approach where the partitioning takes place at the coupling interfaces among models of different dimensions. This allows to parallelize the computations into the sub-domains. However, this splitting strategy, in which the sub-models are solved separately and iteratively, will not be covered here. The monolithic scheme is hereby explored.

\section{NUMERICAL SCHEME}

\subsection{The mesh}

Let us define a mesh of the intervals $\left(\delta, l_{j}\right)$ on the axis $O x^{e_{j}}, j=1, \ldots, n$. For each value of $j$, we choose $N_{j} \in \mathbb{N}^{*}$, and $N_{j}+1$ distinct and increasing values $x_{i+1 / 2}^{e_{j}}, i=0, \ldots, N_{j}$, such that $x_{1 / 2}^{e_{j}}=\delta, x_{N_{j}+1 / 2}^{e_{j}}=l_{j}$. Denote $I_{i}^{e_{j}}=\left(x_{i-1 / 2}^{e_{j}}, x_{i+1 / 2}^{e_{j}}\right)$, and $h_{i}^{e_{j}}=x_{i+1 / 2}^{e_{j}}-x_{i-1 / 2}^{e_{j}}, i=1, \ldots, N_{j}$.

Set $h^{e_{j}}=\max \left\{h_{i}^{e_{j}}, i=1, \ldots, N_{j}\right\}$ the size of the mesh of the interval $\left(\delta, l_{j}\right)$.

Then we choose $N_{j}$ points $x_{i}^{e_{j}}, i=1, \ldots, N_{j}$, such that $x_{i}^{e_{j}} \in I_{i}^{e_{j}}$. Set $x_{0}^{e_{j}}=\delta, x_{N_{j}+1}^{e_{j}}=l_{j}$, and $h_{i+1 / 2}^{e_{j}}=$ $x_{i+1}^{e_{j}}-x_{i}^{e_{j}}, i=0, \ldots, N_{j}$.

Let us construct an admissible mesh over $\Omega_{\varepsilon}^{\prime}$ denoted by $\mathcal{T}$. We assume in the following that $\Omega_{\varepsilon}^{\prime}$ is polygonal. We remind (see the definition in [19]) that such a mesh consists in a family of open polygonal convex subsets $K$ of $\Omega_{\varepsilon}^{\prime}$ (with positive measures) called control volumes, a family of edges $\sigma$ (with strictly positive measures) of the control volumes denoted by $\mathcal{E}$, and a family of points $x_{K}$ chosen in each control volume $K$ denoted by $\mathcal{P}$. The mesh $\mathcal{T}$ satisfies the following properties:

(1) The closure of the union of all the control volumes is $\overline{\Omega_{\varepsilon}^{\prime}}$.

(2) For any $K \in \mathcal{T}$, there is a subset $\mathcal{E}_{K}$ of $\mathcal{E}$ such that $\partial K=\bigcup_{\sigma \in \mathcal{E}_{K}} \bar{\sigma}$, and $\bigcup_{K \in \mathcal{T}} \mathcal{E}_{K}=\mathcal{E}$.

(3) For any $(K, L) \in \mathcal{T}^{2}, K \neq L$, one of three following assertions holds:

either $\bar{K} \cap \bar{L}=\emptyset$, or $\bar{K} \cap \bar{L}$ is a common vertex of $\mathrm{K}$ and $\mathrm{L}$,

or $\bar{K} \cap \bar{L}=\bar{\sigma}, \sigma$ being a common edge of $\mathrm{K}$ and $\mathrm{L}$ denoted by $\sigma_{K / L}$.

(4) The family $\mathcal{P}=\left(x_{K}\right)_{K \in \mathcal{T}}$ is such that for any $K \in \mathcal{T}, x_{K} \in \bar{K}$.

For any $(K, L) \in \mathcal{T}^{2}, K \neq L$, it is assumed that $x_{K} \neq x_{L}$ and that the straight line going

through $x_{K}$ and $x_{L}$ is orthogonal to $\sigma_{K / L}$.

(5) For any $\sigma \in \mathcal{E}$, if $\sigma \subset \partial \Omega_{\varepsilon}^{\prime}, \sigma \in \mathcal{E}_{K}$ and $x_{K} \notin \sigma$, the orthogonal projection of $x_{K}$ on the straight line containing the edge $\sigma$, belongs to $\sigma$. 
Let $\mathcal{E}_{\text {int }}=\left\{\sigma \in \mathcal{E}, \sigma \not \subset \partial \Omega_{\varepsilon}^{\prime}\right\}$.

For any $(K, L) \in \mathcal{T}^{2}, K \neq L$, if $\sigma=\sigma_{K / L}$, let $d_{\sigma}$ be the distance between $x_{K}$ and $x_{L}$. For any $K \in \mathcal{T}$, if $\sigma \in \mathcal{E}_{K}$ and if $\sigma \subset \partial \Omega_{\varepsilon}^{\prime}$, let $d_{\sigma}$ be the distance between $x_{K}$ and $\sigma$.

We assume that for any $\sigma \in \mathcal{E}, d_{\sigma} \neq 0$.

For any $K \in \mathcal{T}$, let $m(K)$ be the area of $K$. For any $\sigma \in \mathcal{E}$, let $m(\sigma)$ be the length of $\sigma$. Let $h_{0}$ be the size of the mesh $\mathcal{T}, h_{0}=\max \{\operatorname{diam}(K), K \in \mathcal{T}\}$, where diam is the abbreviation for diameter.

We denote by $\mathcal{T S}$ the global 1D-2D mesh of $D_{\varepsilon}$. Let $h$ be the size of the 1D-2D mesh of $D_{\varepsilon}: h=$ $\max \left\{h_{0}, h^{e_{j}}, j=1, \ldots, n\right\}$.

\subsection{The hybrid scheme}

The scheme is obtained by integrating $v_{j}^{\prime \prime}=f_{j}$ on each cell $I_{i}^{e_{j}}, i=1, \ldots, N_{j}$, and $\triangle u=0$ over each control volume $K \in \mathcal{T}$. The numerical flux $F_{j, i+1 / 2}$ is an approximation of $v_{j}^{\prime}\left(x_{i+1 / 2}^{e_{j}}\right)$ of finite difference type; $v_{j, i}$ is an approximation of $v_{j}\left(x_{i}^{e_{j}}\right), i=0, \ldots, N_{j}+1$. The flux $F_{K, \sigma}$ through the edge $\sigma$ of the cell $K$ is approximated by a differential quotient. Last $u_{K}$ is an approximation of $u\left(x_{K}\right), K \in \mathcal{T}$. See [36] for details.

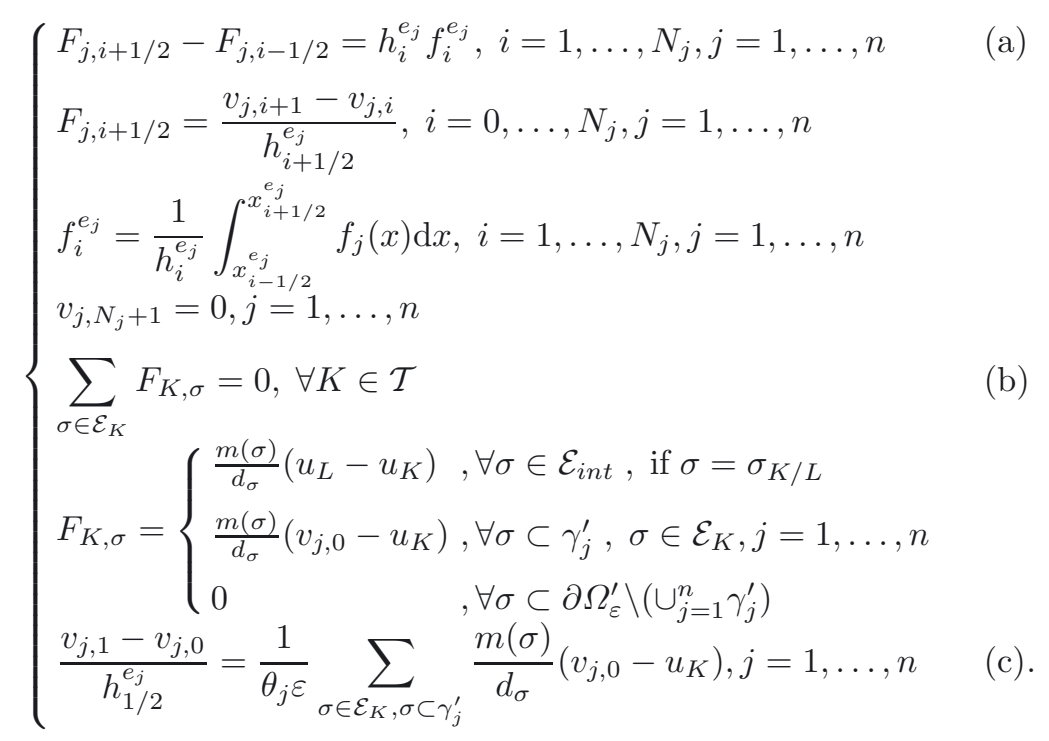

Let us notice that $v_{j, 0}$ is a convex combination of the approximated values of the solution on each side of $\gamma_{j}^{\prime}, j=1, \ldots, n$, since

$$
v_{j, 0}=\left(\frac{v_{j, 1}}{h_{1 / 2}^{e_{j}}}+\frac{1}{\theta_{j} \varepsilon} \sum_{\sigma \in \mathcal{E}_{K}, \sigma \subset \gamma_{j}^{\prime}} \frac{m(\sigma)}{d_{\sigma}} u_{K}\right)\left(\frac{1}{h_{1 / 2}^{e_{j}}}+\frac{1}{\theta_{j} \varepsilon} \sum_{\sigma \subset \gamma_{j}^{\prime}} \frac{m(\sigma)}{d_{\sigma}}\right)^{-1} .
$$

For the sake of simplicity, in (2.2c) and (2.3), the summation is done for $\sigma \subset \gamma_{j}^{\prime}$, and for each of them, $K$ is the control volume such that $\sigma \in \mathcal{E}_{K}$.

The approximate solution of (1.1) is defined by

$$
\begin{gathered}
u_{\mathcal{T}}^{d}(x, y)=\left\{\begin{array}{l}
u_{\mathcal{T}}(x, y),(x, y) \in \Omega_{\varepsilon}^{\prime} \\
v_{j \mathcal{T}}\left(x^{e_{j}}\right),(x, y) \in B_{j}^{\varepsilon}, x^{e_{j}} \in\left(\delta, l_{j}\right), j=1, \ldots, n
\end{array}\right. \\
\text { with }\left\{\begin{array}{l}
u_{\mathcal{T}}(x, y)=u_{K},(x, y) \in K, K \in \mathcal{T} \\
v_{j \mathcal{T}}\left(x^{e_{j}}\right)=v_{j i}, x^{e_{j}} \in\left(x_{i-1 / 2}^{e_{j}}, x_{i+1 / 2}^{e_{j}}\right), i=1, \ldots, N_{j}, j=1, \ldots, n .
\end{array}\right.
\end{gathered}
$$




\subsection{Existence and uniqueness of the finite volume approximation}

The scheme (2.2) leads to a linear system of the form $A U=B$ in which $U$ is the unknown, where $U^{T}=$ $\left(\left\{\left\{v_{j i}, i=1, \ldots, N_{j}\right\}, j=1, \ldots, n\right\},\left\{u_{K}, K \in \mathcal{T}\right\}\right)$.

Lemma 2.1. There is a unique solution $\left(\left\{\left\{v_{j i}, i=1, \ldots, N_{j}\right\}, j=1, \ldots, n\right\},\left\{u_{K}, K \in \mathcal{T}\right\}\right)$ to equations (2.2).

Proof. We assume that $B=0$. Let us prove that $U=0$. We multiply (2.2)a by $v_{j, i}$ and sum over $i$, then multiply by $\theta_{j} \varepsilon$ and sum over $j$. We multiply (2.2)b by $u_{K}$ and sum over K. We obtain

$$
\sum_{j=1}^{n} \theta_{j} \varepsilon \sum_{i=1}^{N_{j}}\left(F_{i+1 / 2}^{j}-F_{i-1 / 2}^{j}\right) v_{j, i}+\sum_{K \in \mathcal{T}} \sum_{\sigma \in \mathcal{E}_{K}} F_{K, \sigma} u_{K}=0 .
$$

Reordering the second summation over the set of edges, we get that

$$
\sum_{j=1}^{n} \theta_{j} \varepsilon\left(\sum_{i=1}^{N_{j}} F_{i+1 / 2}^{j} v_{j, i}-\sum_{i=0}^{N_{j}-1} F_{i+1 / 2}^{j} v_{j, i+1}\right)+\sum_{\substack{\sigma \in \mathcal{E}_{i n t} \\ \sigma=\sigma_{K \mid L}}} F_{K, \sigma}\left(u_{K}-u_{L}\right)+\sum_{j=1}^{n} \sum_{\substack{\sigma \in \mathcal{E}_{K} \\ \sigma \subset \gamma_{j}^{\prime}}} \frac{m(\sigma)}{d_{\sigma}}\left(v_{j, 0}-u_{K}\right) u_{K}=0 .
$$

On the other hand, the definition of the numerical fluxes leads to

$$
\sum_{j=1}^{n} \theta_{j} \varepsilon\left(\sum_{i=1}^{N_{j}}-\frac{\left(v_{j, i+1}-v_{j, i}\right)^{2}}{h_{i+1 / 2}^{e_{j}}}-\frac{v_{j, 1}-v_{j, 0}}{h_{1 / 2}^{e_{j}}} v_{j, 1}\right)-\sum_{\substack{\sigma \in \mathcal{E}_{i n t} \\ \sigma=\sigma_{K} \mid L}} \frac{m(\sigma)}{d_{\sigma}}\left(u_{K}-u_{L}\right)^{2}+\sum_{j=1}^{n} \sum_{\substack{\sigma \in \mathcal{E}_{K} \\ \sigma \subset \gamma_{j}^{\prime}}} \frac{m(\sigma)}{d_{\sigma}}\left(v_{j, 0}-u_{K}\right) u_{K}=0 .
$$

Multiplying (2.2c) by $\theta_{j} \varepsilon v_{j, 0}$, summing over $j$, and adding to the above equality, we get

$$
\sum_{j=1}^{n} \theta_{j} \varepsilon\left(\sum_{i=1}^{N_{j}}-\frac{\left(v_{j, i+1}-v_{j, i}\right)^{2}}{h_{i+1 / 2}^{e_{j}}}-\frac{\left(v_{j, 1}-v_{j, 0}\right)^{2}}{h_{1 / 2}^{e_{j}}}\right)-\sum_{\substack{\sigma \in \mathcal{E}_{i n t} \\ \sigma=\sigma_{K \mid L}}} \frac{m(\sigma)}{d_{\sigma}}\left(u_{K}-u_{L}\right)^{2}-\sum_{j=1}^{n} \sum_{\substack{\sigma \in \mathcal{E}_{K} \\ \sigma \subset \gamma_{j}^{\prime}}} \frac{m(\sigma)}{d_{\sigma}}\left(v_{j, 0}-u_{K}\right)^{2}=0 .
$$

Hence, all the components of $U$ are equal, and since $v_{j, N_{j}+1}=0, j=1, \ldots, n$, we have $U=0$.

Remark 2.2. The previous line reads $-\left\|s_{\mathcal{T}}^{d}\right\|_{1, \mathcal{T}}^{2}=0$ where $\|\cdot\|_{1, \mathcal{T}}$ is defined below (see Def. 3.1), and $s_{\mathcal{T}}^{d}$ is a function constant over each control volume of the mesh $\mathcal{T S}$ which coincides with $u_{\mathcal{T}}^{d}$. The proof of the existence and uniqueness of the solution of (2.2) is also done in [36] using another method.

\section{The Discrete Poincaré inequality}

The proof of an $L^{2}$ error estimate requires a discrete Poincaré inequality. We remind that $D_{\varepsilon}=\Omega_{\varepsilon}^{\prime} \cup\left(\cup_{j=1}^{n} S_{j}\right)$. We introduce the space of piecewise constant functions associated with the 1D-2D mesh of $D_{\varepsilon}$, and a discrete $H^{1}$ norm for this space. The discrete Poincaré inequality, that is established in Lemma 3.3, is expressed in terms of this discrete $H^{1}$ norm.

\section{Definition 3.1.}

(a) We define $X(\mathcal{T})$ the set of functions from $\Omega_{\varepsilon}^{\prime}$ to $\mathbb{R}$ which are constant over each control volume of $\mathcal{T}$.

(b) We define $X(\mathcal{T S})$ the set of functions from $D_{\varepsilon}$ to $\mathbb{R}$ which are constant over each control volume of $\mathcal{T} \mathcal{S}$. 
(c) Let $w \in X(\mathcal{T S})$, such that

$$
w(x, y)=\left\{\begin{array}{l}
w_{K},(x, y) \in K, K \in \mathcal{T} \\
w_{j, i},(x, y) \in S_{j}, x^{e_{j}} \in\left(x_{i-1 / 2}^{e_{j}}, x_{i+1 / 2}^{e_{j}}\right), i=1, \ldots, N_{j}, j=1, \ldots, n .
\end{array}\right.
$$

We define and we denote

(i) $\|w\|_{2, \mathcal{T}}=\left(\sum_{K \in \mathcal{T}} m(K) w_{K}^{2}+\sum_{j=1}^{n} \theta_{j} \varepsilon \sum_{i=1}^{N_{j}} h_{i}^{e_{j}} w_{j, i}^{2}\right)^{1 / 2}$

(ii) $\|w\|_{1, \mathcal{T}, *}=\left(\sum_{\sigma \in \mathcal{E}_{\text {int }}} m(\sigma) d_{\sigma}\left(\frac{D_{\sigma} w}{d_{\sigma}}\right)^{2}\right)^{1 / 2} \quad($ defined also for $w \in X(\mathcal{T}))$

(iii) $\|w\|_{1, \mathcal{T}}=\left(\sum_{\sigma \in \mathcal{E}_{i n t}, \sigma \subset\left(\cup_{j=1}^{n} \gamma_{j}^{\prime}\right)} m(\sigma) d_{\sigma}\left(\frac{D_{\sigma} w}{d_{\sigma}}\right)^{2}+\sum_{j=1}^{n} \theta_{j} \varepsilon \sum_{i=0}^{N_{j}} \frac{\left(w_{j, i+1}-w_{j, i}\right)^{2}}{h_{i+1 / 2}^{e_{j}}}\right)^{1 / 2}$

where $D_{\sigma} w=\left\{\begin{array}{l}\left|w_{K}-w_{L}\right|, \sigma \in \mathcal{E}_{i n t}, \sigma=\sigma_{K \mid L} \\ \left|w_{K}-w_{j, 0}\right|, \sigma \subset \gamma_{j}^{\prime}, \sigma \in \mathcal{E}_{K}, j=1, \ldots, n\end{array}\right.$

$w_{j, N_{j}+1}=0, j=1, \ldots, n$,

and $w_{j, 0}=\left(\frac{w_{j, 1}}{h_{1 / 2}^{e_{j}}}+\frac{1}{\theta_{j} \varepsilon} \sum_{\sigma \in \mathcal{E}_{K}, \sigma \subset \gamma_{j}^{\prime}} \frac{m(\sigma)}{d_{\sigma}} w_{K}\right)\left(\frac{1}{h_{1 / 2}^{e_{j}}}+\frac{1}{\theta_{j} \varepsilon} \sum_{\sigma \subset \gamma_{j}^{\prime}} \frac{m(\sigma)}{d_{\sigma}}\right)^{-1}$.

Remark 3.2. The functions $\|\cdot\|_{2, \mathcal{T}}$ and $\|\cdot\|_{1, \mathcal{T}}$ are norms, and $\|\cdot\|_{1, \mathcal{T}, *}$ is semi-norm, on $X(\mathcal{T S})$. On the other hand, we can explain $\|w\|_{2, \mathcal{T}}$ and $\|w\|_{1, \mathcal{T}}$ as classical discrete norms of a function $\tilde{w}$ defined a.e. on $\Omega_{\varepsilon}$ and such that $\left.\tilde{w}\right|_{D_{\varepsilon}}=w$. Let us define $\tilde{w}$ by

$$
\tilde{w}(x, y)=\left\{\begin{array}{l}
w_{K},(x, y) \in K, K \in \mathcal{T} \\
w_{j, i},(x, y) \in B_{j}^{\varepsilon}, x^{e_{j}} \in\left(x_{i-1 / 2}^{e_{j}}, x_{i+1 / 2}^{e_{j}}\right), i=1, \ldots, N_{j}, j=1, \ldots, n,
\end{array}\right.
$$

then we have $\|w\|_{2, \mathcal{T}}=\|\tilde{w}\|_{L^{2}\left(\Omega_{\varepsilon}\right)}$. We can consider a mesh of $\Omega_{\varepsilon}$ including $\mathcal{T}$ and a row of rectangular cells $\varepsilon$ high by $h$ wide on $B_{j}^{\varepsilon} \backslash B_{j}^{\prime \varepsilon}, j=1, \ldots, n$. The function $\tilde{w}$ is piecewise constant on this mesh, and $\|w\|_{1, \mathcal{T}}$ is equal to a $2 \mathrm{D}$ classical discrete $H^{1}$ norm of $\tilde{w}$ on this mesh.

Lemma 3.3. Let $w \in X(\mathcal{T S})$, there is a constant $c$ independent of $h$ such that

$$
\|w\|_{2, \mathcal{T}}^{2} \leq c\|w\|_{1, \mathcal{T}}^{2}
$$

Proof. Let $w \in X(\mathcal{T S})$ such that

$$
w(x, y)=\left\{\begin{array}{l}
w_{K},(x, y) \in K, K \in \mathcal{T} \\
w_{j, i},(x, y) \in S_{j}, x^{e_{j}} \in\left(x_{i-1 / 2}^{e_{j}}, x_{i+1 / 2}^{e_{j}}\right), i=1, \ldots, N_{j}, j=1, \ldots, n .
\end{array}\right.
$$

We let $w_{j, N_{j}+1}=0, j=1, \ldots, n$, and

$$
w_{j, 0}=\left(\frac{w_{j, 1}}{h_{1 / 2}^{e_{j}}}+\frac{1}{\theta_{j} \varepsilon} \sum_{\sigma \in \mathcal{E}_{K}, \sigma \subset \gamma_{j}^{\prime}} \frac{m(\sigma)}{d_{\sigma}} w_{K}\right)\left(\frac{1}{h_{1 / 2}^{e_{j}}}+\frac{1}{\theta_{j} \varepsilon} \sum_{\sigma \subset \gamma_{j}^{\prime}} \frac{m(\sigma)}{d_{\sigma}}\right)^{-1} .
$$


Noting that

$$
\|w\|_{2, \mathcal{T}}^{2}=\|w\|_{L^{2}\left(\Omega_{\varepsilon}^{\prime}\right)}^{2}+\sum_{j=1}^{n} \theta_{j} \varepsilon \sum_{i=1}^{N_{j}} h_{i}^{e_{j}} w_{j, i}^{2} \leq\|w\|_{L^{2}\left(\Omega_{\varepsilon}^{\prime}\right)}^{2}+\sum_{j=1}^{n} \theta_{j} \varepsilon\left(l_{j}-\delta\right) \sum_{i=0}^{N_{j}} \frac{\left(w_{j, i+1}-w_{j, i}\right)^{2}}{h_{i+1 / 2}^{e_{j}}}
$$

since

$$
\left|w_{j, i}\right| \leq \sum_{i=0}^{N_{j}}\left|w_{j, i}-w_{j, i+1}\right| \leq\left(\sum_{i=0}^{N_{j}} \frac{\left(w_{j, i+1}-w_{j, i}\right)^{2}}{h_{i+1 / 2}^{e_{j}}}\right)^{1 / 2}\left(\sum_{i=0}^{N_{j}} h_{i+1 / 2}^{e_{j}}\right)^{1 / 2}
$$

we deduce that

$$
\|w\|_{2, \mathcal{T}}^{2} \leq\|w\|_{L^{2}\left(\Omega_{\varepsilon}^{\prime}\right)}^{2}+\left(l_{\max }-\delta\right)\|w\|_{1, \mathcal{T}}^{2}
$$

where $l_{\max }=\max \left\{l_{j}, j=1, \ldots, n\right\}$.

Though, proving Lemma 3.3 amounts to proving the existence of a constant $c$ independent of $h$ such that $\|w\|_{L^{2}\left(\Omega_{\varepsilon}^{\prime}\right)}^{2} \leq c\|w\|_{1, \mathcal{T}}^{2}$.

Now, we follow the path of (Lem. 10.2 in [19]) to prove a "discrete mean Poincaré inequality". The authors assume that the domain, in which the problem is set, is an open bounded polygonal connected subset of $\mathbb{R}^{2}$ : $\Omega_{\varepsilon}^{\prime}$ satisfies this requirement allowing the results to be used. Then, following the proof in [19], there is a finite number of disjoint convex polygonal sets, denoted by $\left\{\Omega_{1}, \ldots, \Omega_{p}\right\}$, such that $\bar{\Omega}_{\varepsilon}^{\prime}=\cup_{i=1}^{p} \bar{\Omega}_{i}$. Here, it makes sense to assume that $\Omega_{1}={B^{\prime}}_{1}^{\varepsilon}$ because ${B^{\prime}}_{1}^{\varepsilon}$ is convex, and $\gamma_{1}^{\prime} \subset \partial \Omega_{1}$ is located on the interface. Let $I_{i j}=\bar{\Omega}_{i} \cap \bar{\Omega}_{j}, i \neq j, i, j \in\{1, \ldots, p\}$ as in [19]. Let us remember that only the set of index such that $m\left(I_{i j}\right)>0$ is considered.

Now, let us define the strictly positives quantities $\mu$ and $\lambda$ :

$$
\min \left\{\frac{m\left(I_{i j}\right)}{\varepsilon}, i, j \in\{1, \ldots, p\}\right\}=\mu \quad \min \left\{\frac{m\left(\Omega_{i}\right)}{m\left(\Omega_{\varepsilon}^{\prime}\right)}, i \in\{1, \ldots, p\}\right\}=\lambda .
$$

Why to introduce $\varepsilon$ above to define $\mu$ ? The domain $\Omega_{\varepsilon}$ has been constructed so that the width of each branch is the image of a given segment obtained by a $1 / \varepsilon$-homothetic contraction. Indeed, the thickness of $\Omega_{1}$ is equal to $\theta_{1} \varepsilon$. That is the reason why we do not assume that $m\left(I_{i j}\right)$ is greater than a strictly positive constant (as in [19]), but rather that the ratio $m\left(I_{i j}\right) \varepsilon^{-1}$ is so.

Now, we continue as in [19], defining $m_{1}(w)$ the mean value of $w$ over $\Omega_{1}$, and $m_{\Omega_{\varepsilon}^{\prime}}(w)$ the mean value of $w$ over $\Omega_{\varepsilon}^{\prime}$, that is

$$
m_{1}(w)=\frac{1}{m\left(\Omega_{1}\right)} \int_{\Omega_{1}} w(x, y) \mathrm{d} x \mathrm{~d} y, \quad m_{\Omega_{\varepsilon}^{\prime}}(w)=\frac{1}{m\left(\Omega_{\varepsilon}^{\prime}\right)} \int_{\Omega_{\varepsilon}^{\prime}} w(x, y) \mathrm{d} x \mathrm{~d} y .
$$

Since

$$
\|w\|_{L^{2}\left(\Omega_{\varepsilon}^{\prime}\right)}^{2} \leq 3\left\|w-m_{\Omega_{\varepsilon}^{\prime}}(w)\right\|_{L^{2}\left(\Omega_{\varepsilon}^{\prime}\right)}^{2}+3 m\left(\Omega_{\varepsilon}^{\prime}\right)\left|m_{\Omega_{\varepsilon}^{\prime}}(w)-m_{1}(w)\right|^{2}+3 m\left(\Omega_{\varepsilon}^{\prime}\right) m_{1}(w)^{2}
$$

proving Lemma 3.3 amounts actually to proving the existence of three constants $c_{1}, c_{2}, c_{3}$, independent of $h$ such that
a) $\left\|w-m_{\Omega_{\varepsilon}^{\prime}}(w)\right\|_{L^{2}\left(\Omega_{\varepsilon}^{\prime}\right)}^{2} \leq c_{1}\|w\|_{1, \mathcal{T}}^{2}$
b) $\left|m_{\Omega_{\varepsilon}^{\prime}}(w)-m_{1}(w)\right|^{2} \leq c_{2}\|w\|_{1, \mathcal{T}}^{2}$
c) $m_{1}(w)^{2} \leq c_{3}\|w\|_{1, \mathcal{T}}^{2}$.

The proof of Lemma 10.2 in [19] gives the existence of $c_{1}, c_{2}$, only depending on $\Omega_{\varepsilon}^{\prime}$, such that

$$
\left\|w-m_{\Omega_{\varepsilon}^{\prime}}(w)\right\|_{L^{2}\left(\Omega_{\varepsilon}^{\prime}\right)}^{2} \leq c_{1}\|w\|_{1, \mathcal{T}, *}^{2} \quad\left|m_{\Omega_{\varepsilon}^{\prime}}(w)-m_{1}(w)\right|^{2} \leq c_{2}\|w\|_{1, \mathcal{T}, *}^{2} .
$$

The proof of (3.4a) and (3.4b) follows since $\|w\|_{1, \mathcal{T}, *}^{2} \leq\|w\|_{1, \mathcal{T}}^{2}$. 
Let us prove (3.4c). We consider now the second step of Lemma 10.2 in [19], called "estimate with respect to the mean value on a part of the boundary", for a convex domain. This result is extended in Lemma 7.2 in [43] to the case of meshes where the orthogonality property (2.1)-(4) is not satisfied. Similarly, Lemma 2.7.2 in [44] gives a result for functions which are null on a part of the boundary, this proof is an alternative to the second step of Lemma 10.2 in [19] and it is easily applied in the current context. That is why we follow now the proof in [44], taking $\Omega_{1}$ for the convex domain and $\gamma_{1}^{\prime} \subset \partial \Omega_{1}$ for the part of the boundary with a null Dirichlet condition. Of course, the function $w$ is not null on $\gamma_{1}^{\prime}$. It is the difference between the result obtained in [19] or [44], and Lemma 3.3. Introducing $\|w\|_{1, \mathcal{T}}^{2}$ instead of $\|w\|_{1, \mathcal{T}_{*}}^{2}$ allows to overcome this difficulty.

As in [44], we begin the proof of (3.4c) by choosing a vector $b_{1}$, such that, for each point in $\Omega_{1}$, each line defined by this point and $b_{1}$ intersects $\gamma_{1}^{\prime}$. We take $b_{1}=e_{1}$. We need here only one vector, while the author need a family of vectors in [44]. Now, we adapt this proof to our geometrical multi-scale domain.

For all $(x, y) \in \Omega_{1}, D\left((x, y), e_{1}\right)$ designates the semi-line defined by its origin $(x, y)$ and the vector $e_{1}$; let $P(x, y)=\gamma_{1}^{\prime} \cap D\left((x, y), e_{1}\right)$.

For $\sigma \in \mathcal{E}, \chi_{\sigma}$ is a function from $\mathbb{R}^{2} \times \mathbb{R}^{2}$ to $\{0,1\}$ such that $\chi_{\sigma}(r, z)$ is equal to 1 if $\sigma \cap[r, z] \neq \emptyset$ and equal to 0 otherwise.

Let $K \in \mathcal{T}$ such that $K \cap \Omega_{1} \neq \emptyset$. Then we have for a.e. $(x, y) \in K \cap \Omega_{1}$ :

$$
\left|w_{K}\right| \leq \sum_{\sigma \in \mathcal{E}_{i n t}, \sigma \subset \gamma_{1}^{\prime}}\left(D_{\sigma} w\right) \chi_{\sigma}((x, y), P(x, y))+\sum_{i=0}^{N_{1}}\left|w_{1, i}-w_{1, i+1}\right|
$$

since $w_{1, N_{1}+1}=0$. This requirement is essential to ensure the inequality above. Let us remark that there is $\sigma \subset \gamma_{1}^{\prime}$ such that $P(x, y) \in \sigma$, then $D_{\sigma} w=\left|w_{L}-w_{1,0}\right|$ for some $L$ (see Def. 3.1) such that $\sigma \in \mathcal{E}_{L}$. The use of $w_{1,0}$ allows to get out of $\Omega_{\varepsilon}^{\prime}$ and join the boundary of the $1 \mathrm{D}$ domain $S_{1}$.

By the Cauchy-Schwarz inequality, we have

$$
w_{K}^{2} \leq\left(\sum_{\substack{\sigma \in \mathcal{E}_{\text {int }} \\ \sigma \subset \gamma_{1}^{\prime}}} \frac{\left(D_{\sigma} w\right)^{2}}{d_{\sigma} c_{\sigma}} \chi_{\sigma}((x, y), P(x, y))+\sum_{i=0}^{N_{1}} \frac{\left(w_{1, i}-w_{1, i+1}\right)^{2}}{h_{i+1 / 2}^{e_{1}}}\right)\left(\sum_{\substack{\sigma \in \mathcal{E}_{\text {int }} \\ \sigma \subset \gamma_{1}^{\prime}}} d_{\sigma} c_{\sigma} \chi_{\sigma}((x, y), P(x, y))+\sum_{i=0}^{N_{1}} h_{i+1 / 2}^{e_{j}}\right)
$$

where $c_{\sigma}=\left|e_{1} \cdot n_{\sigma}\right|$.

Since $e_{1}$ is the axis of the first branch (where $\Omega_{1}$ is found), we have

$$
\sum_{\sigma \in \mathcal{E}_{i n t}, \sigma \subset \gamma_{1}^{\prime}} d_{\sigma} c_{\sigma} \chi_{\sigma}((x, y), P(x, y)) \leq \delta
$$

Integrating (3.5) over $K \cap \Omega_{1}$ and summing over all $K \in \mathcal{T}$ such that $K \cap \Omega_{1} \neq \emptyset$ yields

$$
\sum_{K \in \mathcal{T}} w_{K}^{2} m\left(K \cap \Omega_{1}\right) \leq l_{1}\left(\sum_{\substack{\sigma \in \mathcal{E}_{\text {int }} \\ \sigma \subset \gamma_{1}^{\prime}}} \frac{\left(D_{\sigma} w\right)^{2}}{d_{\sigma} c_{\sigma}}\left(\int_{\Omega_{1}} \chi_{\sigma}((x, y), P(x, y)) \mathrm{d} x \mathrm{~d} y\right)+m\left(\Omega_{1}\right) \sum_{i=0}^{N_{1}} \frac{\left(w_{1, i}-w_{1, i+1}\right)^{2}}{h_{i+1 / 2}^{e_{1}}}\right) .
$$

Since, following [19], we have

$$
\int_{\Omega_{1}} \chi_{\sigma}((x, y), P(x, y)) \mathrm{d} x \mathrm{~d} y \leq \delta m(\sigma) c_{\sigma}
$$


then (3.6) implies that

$$
\begin{aligned}
\|w\|_{L^{2}\left(\Omega_{1}\right)}^{2} & \leq l_{\max }\left(\delta \sum_{\sigma \in \mathcal{E}_{\text {int }}, \sigma \subset \gamma_{1}^{\prime}} m(\sigma) d_{\sigma}\left(\frac{D_{\sigma} w}{d_{\sigma}}\right)^{2}+m\left(\Omega_{1}\right) \sum_{i=0}^{N_{1}} \frac{\left(w_{1, i}-w_{1, i+1}\right)^{2}}{h_{i+1 / 2}^{e_{1}}}\right) \\
& \leq l_{\max } \delta\left(\sum_{\sigma \in \mathcal{E}_{\text {int }, \sigma \subset \gamma_{1}^{\prime}}} m(\sigma) d_{\sigma}\left(\frac{D_{\sigma} w}{d_{\sigma}}\right)^{2}+\theta_{1} \varepsilon \sum_{i=0}^{N_{1}} \frac{\left(w_{1, i}-w_{1, i+1}\right)^{2}}{h_{i+1 / 2}^{e_{1}}}\right) \\
& \leq l_{\max } \delta\|w\|_{1, \mathcal{T}}^{2} .
\end{aligned}
$$

As we have

$$
m_{1}(w)^{2} \leq \frac{1}{m\left(\Omega_{1}\right)}\|w\|_{L^{2}\left(\Omega_{1}\right)}^{2} \leq \frac{l_{\max } \delta}{m\left(\Omega_{1}\right)}\|w\|_{1, \mathcal{T}}^{2}
$$

this proves $(3.4 \mathrm{c})$.

With (3.1) and (3.3), we deduce that there is a constant $c$ depending only on $D_{\varepsilon}$ such that $\|w\|_{2, \mathcal{T}}^{2} \leq c\|w\|_{1, \mathcal{T}}^{2}$, so Lemma 3.3 is proved. This lemma is used to state Theorems 4.1 and 4.4 below. Theorem 4.1 gives an error estimate for (1.1) assuming $\varepsilon$ and $\delta$ are fixed. Theorem 4.4 relates to (1.3) assuming $\varepsilon$ tends to zero.

If we are just interested in the resolution of (1.1) then a more precise definition of the constant $c$ does not matter. To get the estimate of Theorem 4.1 it is enough to know that $c$ depends only on $D_{\varepsilon}$.

The estimate of Theorem 4.4 requires precise informations on the dependence of $c_{1}, c_{2}, c_{3}$ with respect to $\varepsilon$ and $\delta$. Evaluating the constants from the proof of Lemma 10.2 in [19], one has

$$
\begin{aligned}
& c_{1}=O\left(\frac{\operatorname{diam}\left(\Omega_{\varepsilon}^{\prime}\right)^{4} m\left(\Omega_{k}\right)}{m\left(\Omega_{i}\right)^{2}}+\frac{\operatorname{diam}\left(\Omega_{\varepsilon}^{\prime}\right) \operatorname{diam}\left(\Omega_{i}\right)^{2} m\left(\Omega_{k}\right)}{m\left(I_{i j}\right) m\left(\Omega_{i}\right)}+\frac{\operatorname{diam}\left(\Omega_{\varepsilon}^{\prime}\right)^{4}}{m\left(\Omega_{i}\right)}, i, j, k \in\{1, \ldots, p\}\right) \\
& c_{2}=O\left(\frac{\operatorname{diam}\left(\Omega_{\varepsilon}^{\prime}\right)^{4}}{m\left(\Omega_{i}\right)^{2}}+\frac{\operatorname{diam}\left(\Omega_{\varepsilon}^{\prime}\right) \operatorname{diam}\left(\Omega_{i}\right)^{2}}{m\left(I_{i j}\right) m\left(\Omega_{i}\right)}, i, j \in\{1, \ldots, p\}\right) .
\end{aligned}
$$

We remind that we assume in this case that $\delta$ is of order $\varepsilon \ln \varepsilon$. With (3.2), we deduce that

$$
c_{1}=O\left(\frac{\operatorname{diam}\left(\Omega_{\varepsilon}^{\prime}\right)^{4}}{m\left(\Omega_{\varepsilon}^{\prime}\right)}+\frac{\operatorname{diam}\left(\Omega_{\varepsilon}^{\prime}\right)^{3}}{\varepsilon}\right)=O\left(\frac{\delta^{3}}{\varepsilon}\right) \quad c_{2}=O\left(\frac{\operatorname{diam}\left(\Omega_{\varepsilon}^{\prime}\right)^{4}}{m\left(\Omega_{\varepsilon}^{\prime}\right)^{2}}+\frac{\operatorname{diam}\left(\Omega_{\varepsilon}^{\prime}\right)^{3}}{\varepsilon m\left(\Omega_{\varepsilon}^{\prime}\right)}\right)=O\left(\frac{\delta^{2}}{\varepsilon^{2}}\right) .
$$

Last we have

$$
c_{3}=\frac{l_{\max } \delta}{m\left(\Omega_{1}\right)}=O\left(\frac{1}{\varepsilon}\right)
$$

And then, we see from (3.1), (3.3) and (3.4) that there is a constant $c$, namely

$$
c=3\left(c_{1}+m\left(\Omega_{\varepsilon}^{\prime}\right) c_{2}+m\left(\Omega_{\varepsilon}^{\prime}\right) c_{3}\right)+l_{\max }-\delta
$$

such that

$$
\|w\|_{2, \mathcal{T}}^{2} \leq c\|w\|_{1, \mathcal{T}}^{2}
$$

Moreover, we conclude with (3.8) and (3.9) that

$$
c=O\left(\frac{\delta^{3}}{\varepsilon}\right)+O(\delta)+O(1)=O(1)
$$

when $\varepsilon$ tends to zero, assuming that $\delta$ is of order $\varepsilon \ln (\varepsilon)$. So, also in this case, the constant $c$ in Lemma 3.3 depends neither on $h$ nor on $\varepsilon$. 


\section{The ERRor ESTIMATE}

The error estimate between the solution of (1.1) and its finite volume approximation, which is obtained in [36], uses the linearity of the problem to prevent the coupling between its 1D and its 2D parts. So in [36], a standard $H^{1}$ norm on the $1 \mathrm{D}$ domains $S_{j}, j=1, \ldots, n$, and a standard $H^{1}$ norm on the 2D domain $\Omega_{\varepsilon}^{\prime}$ are used. The disadvantage of this method is that the errors between the values $\alpha_{j}, j=1, \ldots, n$, of the solution on the interfaces between the domains of different dimensions and the approximate values $v_{j, 0}$, play an important role in calculating the global error. And these errors are not optimized (see Sect. 1.6).

To overcome this difficulty, we use here the specific discrete $H^{1}$ norm defined in the previous section on $D_{\varepsilon}$. Using (2.3), the approximate values $v_{j, 0}, j=1, \ldots, n$, of the solution on the interfaces are related to (convex combinations of) the other unknowns: the approximate values of the solution on both sides of the interfaces between the $1 \mathrm{D}$ parts and the $2 \mathrm{D}$ part. So $v_{j, 0}, j=1, \ldots, n$, may be removed from the scheme (2.2) by expressing $v_{j, 0}$ in terms of $v_{j, 1}$ and $u_{K}$ such that there is $\sigma \in \mathcal{E}_{K}, \sigma \subset \gamma_{j}^{\prime}$, according to (2.3). In the same way, $\|w\|_{1, \mathcal{T}}$ may be rewritten without $w_{j, 0}, j=1, \ldots, n$, in Definition 3.1. The global error $e_{\mathcal{T}}$ is defined just below, an estimate of $\left\|e_{\mathcal{T}}\right\|_{1, \mathcal{T}}$ is obtained without using any estimate on $\left|\alpha_{j}-v_{j, 0}\right|, j=1, \ldots, n$, that allows to improve the result obtained in [36].

We remind that the solution of (1.1) is assumed to be regular, that means that $u \in C^{2}\left(\overline{\Omega_{\varepsilon}^{\prime}}\right)$ and $v_{j} \in$ $C^{2}\left(\left[\delta, l_{j}\right]\right), j=1, \ldots, n$.

We state below the main result of the paper.

Theorem 4.1. If $u_{\mathcal{T}}^{d}$ is the finite volume approximation of (1.1) defined by (2.4), if $u^{d}$ is the solution of (1.1) defined by (1.2) and is assumed to be regular, and if $e_{\mathcal{T}} \in X(\mathcal{T S})$ is defined by

$$
e_{\mathcal{T}}(x, y)=\left\{\begin{array}{l}
e_{K}=u\left(x_{K}\right)-u_{K},(x, y) \in K, K \in \mathcal{T} \\
e_{j, i}=v_{j}\left(x_{i}^{e_{j}}\right)-v_{j, i},(x, y) \in S_{j}, x^{e_{j}} \in\left(x_{i-1 / 2}^{e_{j}}, x_{i+1 / 2}^{e_{j}}\right), i=1, \ldots, N_{j}, j=1, \ldots, n .
\end{array}\right.
$$

and if we let

$$
\begin{aligned}
& e_{j, 0}=\left(\frac{e_{j, 1}}{h_{1 / 2}^{e_{j}}}+\frac{1}{\theta_{j} \varepsilon} \sum_{\sigma \in \mathcal{E}_{K}, \sigma \subset \gamma_{j}^{\prime}} \frac{m(\sigma)}{d_{\sigma}} e_{K}\right)\left(\frac{1}{h_{1 / 2}^{e_{j}}}+\frac{1}{\theta_{j} \varepsilon} \sum_{\sigma \subset \gamma_{j}^{\prime}} \frac{m(\sigma)}{d_{\sigma}}\right)^{-1} \\
& e_{j, N_{j}+1}=0, j=1, \ldots, n
\end{aligned}
$$

then, there are two constants $c_{1}$ and $c_{2}$ depending only on $u^{d}$ and $D_{\varepsilon}$ such that

$$
\left\|e_{\mathcal{T}}\right\|_{1, \mathcal{T}} \leq c_{1} h
$$

and

$$
\left\|u^{d}-u_{\mathcal{T}}^{d}\right\|_{L^{2}\left(\Omega_{\varepsilon}\right)} \leq c_{2} h
$$

with $h$ the size of the mesh of $D_{\varepsilon}$.

Proof. We prove an estimate for $\left\|e_{\mathcal{T}}\right\|_{1, \mathcal{T}}$, and conclude thanks to the Poincaré inequality. This proof is not classical because of the interface terms relating to the consistency error on the diffusion flux when $\sigma \subset \gamma_{j}^{\prime}, j=$ $1, \ldots, n$.

We consider first the continuous problem (1.1). We integrate (1.1a) over each 1D cell and (1.1b) over each $K \in \mathcal{T}$. We obtain

$$
\left\{\begin{array}{l}
\bar{F}_{j, i+1 / 2}-\bar{F}_{j, i-1 / 2}=h_{i}^{e_{j}} f_{i}^{e_{j}}, i=1, \ldots, N_{j}, j=1, \ldots, n \\
\bar{F}_{j, i+1 / 2}=v_{j}^{\prime}\left(x_{i+1 / 2}^{e_{j}}\right), i=0, \ldots, N_{j}, j=1, \ldots, n \\
\sum_{\sigma \in \mathcal{E}_{K}} \bar{F}_{K, \sigma}=0, \forall K \in \mathcal{T} \\
\bar{F}_{K, \sigma}=\int_{\sigma} \frac{\partial u}{\partial n} \mathrm{~d} \gamma, \forall \sigma \in \mathcal{E}_{K} .
\end{array}\right.
$$


We define

$$
\left\{\begin{array}{l}
F_{j, i+1 / 2}^{*}=\frac{v_{j}\left(x_{i+1}^{e_{j}}\right)-v_{j}\left(x_{i}^{e_{j}}\right)}{h_{i+1 / 2}^{e_{j}}}, i=1, \ldots, N_{j}, j=1, \ldots, n \\
F_{j, 1 / 2}^{*}=\frac{v_{j}\left(x_{1}^{e_{j}}\right)-u_{j}^{*}(\delta)}{h_{1 / 2}^{e_{j}}}, j=1, \ldots, n
\end{array}\right.
$$

with

$$
u_{j}^{*}(\delta)=\left(\frac{v_{j}\left(x_{1}^{e_{j}}\right)}{h_{1 / 2}^{e_{j}}}+\frac{1}{\theta_{j} \varepsilon} \sum_{\sigma \in \mathcal{E}_{K}, \sigma \subset \gamma_{j}^{\prime}} \frac{m(\sigma)}{d_{\sigma}} u\left(x_{K}\right)\right)\left(\frac{1}{h_{1 / 2}^{e_{j}}}+\frac{1}{\theta_{j} \varepsilon} \sum_{\sigma \subset \gamma_{j}^{\prime}} \frac{m(\sigma)}{d_{\sigma}}\right)^{-1}, j=1, \ldots, n .
$$

In the same spirit, we introduce

$$
F_{K, \sigma}^{*}= \begin{cases}\frac{m(\sigma)}{d_{\sigma}}\left(u\left(x_{L}\right)-u\left(x_{K}\right)\right), & \forall \sigma \in \mathcal{E}_{i n t}, \text { if } \sigma=\sigma_{K / L} \\ \frac{m(\sigma)}{d_{\sigma}}\left(u_{j}^{*}(\delta)-u\left(x_{K}\right)\right), & \forall \sigma \subset \gamma_{j}^{\prime}, \sigma \in \mathcal{E}_{K}, j=1, \ldots, n \\ 0, & \forall \sigma \subset \partial \Omega_{\varepsilon}^{\prime} \backslash\left(\cup_{j=1}^{n} \gamma_{j}^{\prime}\right) .\end{cases}
$$

The consistency errors are defined by

$$
\left\{\begin{aligned}
R_{j, i+1 / 2} & =F_{j, i+1 / 2}^{*}-\bar{F}_{j, i+1 / 2}, i=0, \ldots, N_{j}, j=1, \ldots, n \\
R_{K, \sigma} & =\frac{1}{m(\sigma)}\left(F_{K, \sigma}^{*}-\bar{F}_{K, \sigma}\right), \forall \sigma \in \mathcal{E}_{K}, \forall K \in \mathcal{T}
\end{aligned}\right.
$$

We have

$$
\left\{\begin{array}{l}
R_{j, i+1 / 2}=O\left(h\left\|v_{j}^{\prime \prime}\right\|_{\infty}\right), i=1, \ldots, N_{j}, j=1, \ldots, n \\
R_{K, \sigma}=O\left(h\left\|\nabla^{2} u\right\|_{\infty}\right), \forall \sigma \in \mathcal{E}_{K} \cap \mathcal{E}_{i n t}, \forall K \in \mathcal{T} \\
R_{K, \sigma}=0, \forall \sigma \in \mathcal{E}_{K}, \forall \sigma \subset \partial \Omega_{\varepsilon}^{\prime} \backslash\left(\cup_{j=1}^{n} \gamma_{j}^{\prime}\right), \forall K \in \mathcal{T}
\end{array}\right.
$$

Now, in order to deal with the consistency errors at the interfaces, we define the following quantities for all $j=1, \ldots, n$ and $\sigma \in \mathcal{E}_{K}, \sigma \subset \gamma_{j}^{\prime}$

$$
\left\{\begin{array}{l}
R_{j, 1 / 2}^{*}=\frac{v_{j}(\delta)-u_{j}^{*}(\delta)}{h_{1 / 2}^{e_{j}}}, R_{j, 1 / 2}^{\nabla}=\frac{v_{j}\left(x_{1}^{e_{j}}\right)-v_{j}(\delta)}{h_{1 / 2}^{e_{j}}}-v_{j}^{\prime}(\delta) \\
R_{K, \sigma}^{*}=\frac{u_{j}^{*}(\delta)-v_{j}(\delta)}{d_{\sigma}}, R_{K, \sigma}^{\nabla}=\frac{v_{j}(\delta)-u\left(x_{K}\right)}{d_{\sigma}}-\frac{1}{m(\sigma)} \int_{\sigma} \frac{\partial u}{\partial n} \mathrm{~d} \gamma
\end{array}\right.
$$

We have for all $j=1, \ldots, n$ and $\sigma \in \mathcal{E}_{K}, \sigma \subset \gamma_{j}^{\prime}$

$$
R_{j, 1 / 2}^{\nabla}=O\left(h\left\|v_{j}^{\prime \prime}\right\|_{\infty}\right), R_{K, \sigma}^{\nabla}=O\left(h\left\|\nabla^{2} u\right\|_{\infty}\right) .
$$

We let for all $j=1, \ldots, n$ and $\sigma \in \mathcal{E}_{K}, \sigma \subset \gamma_{j}^{\prime}$

$$
R_{j, 1 / 2}=R_{j, 1 / 2}^{*}+R_{j, 1 / 2}^{\nabla}, R_{K, \sigma}=R_{K, \sigma}^{*}+R_{K, \sigma}^{\nabla} .
$$

Now, we prove the following intermediate lemma

Lemma 4.2. $\sum_{\sigma \in \mathcal{E}_{K}, \sigma \subset \gamma_{j}^{\prime}} m(\sigma) R_{K, \sigma}-\theta_{j} \varepsilon R_{j, 1 / 2}=0, j=1, \ldots, n$. 
Proof. The summation above is done for $\sigma \subset \gamma_{j}^{\prime}$, and for each of them, $K$ is the control volume such that $\sigma \in \mathcal{E}_{K}$ (as in (2.2c) and (2.3)). We have

$$
\begin{aligned}
& \sum_{\sigma \in \mathcal{E}_{K}, \sigma \subset \gamma_{j}^{\prime}} m(\sigma) R_{K, \sigma}-\theta_{j} \varepsilon R_{j, 1 / 2} \\
= & \sum_{\sigma \in \mathcal{E}_{K}, \sigma \subset \gamma_{j}^{\prime}}\left(\frac{m(\sigma)}{d_{\sigma}}\left(u_{j}^{*}(\delta)-u\left(x_{K}\right)\right)-\int_{\sigma} \frac{\partial u}{\partial n} \mathrm{~d} \gamma\right)-\theta_{j} \varepsilon\left(\frac{v_{j}\left(x_{1}^{e_{j}}\right)-u_{j}^{*}(\delta)}{h_{1 / 2}^{e_{j}}}-v_{j}^{\prime}(\delta)\right) \\
= & u_{j}^{*}(\delta) \theta_{j} \varepsilon\left(\frac{1}{h_{1 / 2}^{e_{j}}}+\frac{1}{\theta_{j} \varepsilon} \sum_{\sigma \subset \gamma_{j}^{\prime}} \frac{m(\sigma)}{d_{\sigma}}\right)-\theta_{j} \varepsilon\left(\frac{v_{j}\left(x_{1}^{e_{j}}\right)}{h_{1 / 2}^{e_{j}}}+\frac{1}{\theta_{j} \varepsilon} \sum_{\substack{\sigma \in \mathcal{E}_{K} \\
\sigma \subset \gamma_{j}^{\prime}}} \frac{m(\sigma)}{d_{\sigma}} u\left(x_{K}\right)\right)+\theta_{j} \varepsilon v_{j}^{\prime}(\delta)-\sum_{\sigma \subset \gamma_{j}^{\prime}} \int_{\sigma} \frac{\partial u}{\partial n} \mathrm{~d} \gamma .
\end{aligned}
$$

We conclude with (1.1c) and (4.5) that

$$
\sum_{\sigma \in \mathcal{E}_{K}, \sigma \subset \gamma_{j}^{\prime}} m(\sigma) R_{K, \sigma}-\theta_{j} \varepsilon R_{j, 1 / 2}=0, j=1, \ldots, n .
$$

Now to continue the proof of Theorem 4.1, we substract equations of (2.2) and (4.3) one by one, and obtain

$$
\left\{\begin{array}{l}
\bar{F}_{j, i+1 / 2}-F_{j, i+1 / 2}-\left(\bar{F}_{j, i-1 / 2}-F_{j, i-1 / 2}\right)=0, i=1, \ldots, N_{j}, j=1, \ldots, n \\
\sum_{\sigma \in \mathcal{E}_{K}}\left(\bar{F}_{K, \sigma}-F_{K, \sigma}\right)=0, \forall K \in \mathcal{T} .
\end{array}\right.
$$

Then we introduce the consistency errors. With (4.7) we get

$$
\left\{\begin{array}{l}
F_{j, i+1 / 2}^{*}-F_{j, i+1 / 2}-\left(F_{j, i-1 / 2}^{*}-F_{j, i-1 / 2}\right)-\left(R_{j, i+1 / 2}-R_{j, i-1 / 2}\right)=0, i=1, \ldots, N_{j}, j=1, \ldots, n \\
\sum_{\sigma \in \mathcal{E}_{K}}\left(F_{K, \sigma}^{*}-F_{K, \sigma}\right)-\sum_{\sigma \in \mathcal{E}_{K}} m(\sigma) R_{K, \sigma}=0, \forall K \in \mathcal{T} .
\end{array}\right.
$$

We remark that $e_{j, 0}=u_{j}^{*}(\delta)-v_{j, 0}$ (the definition of $v_{j, 0}$ is given in $(2.3)$ ).

Since $e_{j, N_{j}+1}=0, j=1, \ldots, n$, we thus have

$$
\left\{\begin{array}{l}
F_{j, i+1 / 2}^{*}-F_{j, i+1 / 2}=\frac{e_{j, i+1}-e_{j, i}}{h_{i+1 / 2}^{e_{j}}}, i=1, \ldots, N_{j}, j=1, \ldots, n \\
F_{j, 1 / 2}^{*}-F_{j, 1 / 2}=\frac{e_{j, 1}-e_{j, 0}}{h_{1 / 2}^{e_{j}}}, i=1, \ldots, N_{j}, j=1, \ldots, n \\
F_{K, \sigma}^{*}-F_{K, \sigma}= \begin{cases}\frac{m(\sigma)}{d_{\sigma}}\left(e_{L}-e_{K}\right) & , \forall \sigma \in \mathcal{E}_{i n t}, \text { if } \sigma=\sigma_{K / L} \\
\frac{m(\sigma)}{d_{\sigma}}\left(e_{j, 0}-e_{K}\right) & , \forall \sigma \subset \gamma_{j}^{\prime}, \sigma \in \mathcal{E}_{K}, j=1, \ldots, n \\
0 & , \forall \sigma \subset \partial \Omega_{\varepsilon}^{\prime} \backslash\left(\cup_{j=1}^{n} \gamma_{j}^{\prime}\right) .\end{cases}
\end{array}\right.
$$

Using the above expressions in (4.13), we get

$$
\left\{\begin{array}{l}
\frac{e_{j, i+1}-e_{j, i}}{h_{i+1 / 2}^{e_{j}}}-\frac{e_{j, i}-e_{j, i-1}}{h_{i-1 / 2}^{e_{j}}}=R_{j, i+1 / 2}-R_{j, i-1 / 2}, i=1, \ldots, N_{j}, j=1, \ldots, n \\
\sum_{\sigma \in \mathcal{E}_{K} \cap \mathcal{E}_{i n t}} \frac{m(\sigma)}{d_{\sigma}}\left(e_{L}-e_{K}\right)+\sum_{\sigma \in \mathcal{E}_{K}} \sum_{j=1}^{n} \sum_{\sigma \subset \gamma_{j}^{\prime}} \frac{m(\sigma)}{d_{\sigma}}\left(e_{j, 0}-e_{K}\right)=\sum_{\sigma \in \mathcal{E}_{K}} m(\sigma) R_{K, \sigma}, \forall K \in \mathcal{T} \text { (b). }
\end{array}\right.
$$


Multiplying (4.14a) by $e_{j, i}$, summing over $i$, we obtain

$$
-\sum_{i=1}^{N_{j}} \frac{\left(e_{j, i+1}-e_{j, i}\right)^{2}}{h_{i+1 / 2}^{e_{j}}}-\frac{e_{j, 1}-e_{j, 0}}{h_{1 / 2}^{e_{j}}} e_{j, 1}=\sum_{i=1}^{N_{j}} R_{j, i+1 / 2}\left(e_{j, i}-e_{j, i+1}\right)-R_{j, 1 / 2} e_{j, 1},, j=1, \ldots, n .
$$

Multiplying (4.14b) by $e_{K}$, summing over $K$, we obtain

$$
\begin{aligned}
-\sum_{\sigma \in \mathcal{E}_{i n t}, \sigma=\sigma_{K / L}} \frac{m(\sigma)}{d_{\sigma}}\left(e_{L}-e_{K}\right)^{2}+ & \sum_{j=1}^{n} \sum_{\sigma \in \mathcal{E}_{K}, \sigma \subset \gamma_{j}^{\prime}} \frac{m(\sigma)}{d_{\sigma}}\left(e_{j, 0}-e_{K}\right) e_{K} \\
& =\sum_{\sigma \in \mathcal{E}_{i n t}, \sigma=\sigma_{K / L}} m(\sigma) R_{K, \sigma}\left(e_{K}-e_{L}\right)+\sum_{j=1}^{n} \sum_{\sigma \in \mathcal{E}_{K}, \sigma \subset \gamma_{j}^{\prime}} m(\sigma) R_{K, \sigma} e_{K} .
\end{aligned}
$$

The right summations (in each member) are done for $\sigma \subset \gamma_{j}^{\prime}$, and for each of them, $K$ is the control volume such that $\sigma \in \mathcal{E}_{K}$ (as in (2.2c) and (2.3)). We multiply (4.15) by $\theta_{j} \varepsilon$, sum over $j$, and add (4.16). Then we consider the two quantities with terms on the interfaces. The first one, depending on $e_{j, 0}$, may be rewritten

$$
-\theta_{j} \varepsilon \frac{e_{j, 1}-e_{j, 0}}{h_{1 / 2}^{e_{j}}} e_{j, 1}+\sum_{\sigma \in \mathcal{E}_{K}, \sigma \subset \gamma_{j}^{\prime}} \frac{m(\sigma)}{d_{\sigma}}\left(e_{j, 0}-e_{K}\right) e_{K}=-\theta_{j} \varepsilon \frac{\left(e_{j, 1}-e_{j, 0}\right)^{2}}{h_{1 / 2}^{e_{j}}}-\sum_{\sigma \in \mathcal{E}_{K}, \sigma \subset \gamma_{j}^{\prime}} \frac{m(\sigma)}{d_{\sigma}}\left(e_{j, 0}-e_{K}\right)^{2}
$$

because

$$
\frac{e_{j, 1}-e_{j, 0}}{h_{1 / 2}^{e_{j}}}=\frac{1}{\theta_{j} \varepsilon} \sum_{\sigma \in \mathcal{E}_{K}, \sigma \subset \gamma_{j}^{\prime}} \frac{m(\sigma)}{d_{\sigma}}\left(e_{j, 0}-e_{K}\right), j=1, \ldots, n
$$

The second one, depending on $R_{j, 1 / 2}$ and $R_{K, \sigma}$ with $\sigma \in \mathcal{E}_{K}, \sigma \subset \gamma_{j}^{\prime}$, can be written thanks to Lemma 5.2,

$$
-\theta_{j} \varepsilon R_{j, 1 / 2} e_{j, 1}+\sum_{\sigma \in \mathcal{E}_{K}, \sigma \subset \gamma_{j}^{\prime}} m(\sigma) R_{K, \sigma} e_{K}=-\theta_{j} \varepsilon R_{j, 1 / 2}\left(e_{j, 1}-e_{j, 0}\right)+\sum_{\sigma \in \mathcal{E}_{K}, \sigma \subset \gamma_{j}^{\prime}} m(\sigma) R_{K, \sigma}\left(e_{K}-e_{j, 0}\right)
$$

then we use (4.18) again, which implies that

$$
\begin{aligned}
-\theta_{j} \varepsilon R_{j, 1 / 2}^{*}\left(e_{j, 1}-e_{j, 0}\right) & +\sum_{\sigma \in \mathcal{E}_{K}, \sigma \subset \gamma_{j}^{\prime}} m(\sigma) R_{K, \sigma}^{*}\left(e_{K}-e_{j, 0}\right) \\
& =\left(u_{j}^{*}(\delta)-v_{j}(\delta)\right)\left(\theta_{j} \varepsilon \frac{e_{j, 1}-e_{j, 0}}{h_{1 / 2}^{e_{j}}}+\sum_{\sigma \in \mathcal{E}_{K}, \sigma \subset \gamma_{j}^{\prime}} \frac{m(\sigma)}{d_{\sigma}}\left(e_{K}-e_{j, 0}\right)\right)
\end{aligned}
$$

and allows to simplify (4.19) in the following way

$$
-\theta_{j} \varepsilon R_{j, 1 / 2} e_{j, 1}+\sum_{\sigma \in \mathcal{E}_{K}, \sigma \subset \gamma_{j}^{\prime}} m(\sigma) R_{K, \sigma} e_{K}=-\theta_{j} \varepsilon R_{j, 1 / 2}^{\nabla}\left(e_{j, 1}-e_{j, 0}\right)+\sum_{\sigma \in \mathcal{E}_{K}, \sigma \subset \gamma_{j}^{\prime}} m(\sigma) R_{K, \sigma}^{\nabla}\left(e_{K}-e_{j, 0}\right) .
$$


So multiplying (4.15) by $\theta_{j} \varepsilon$, summing over $j$, adding (4.16), using (4.17) and (4.20), we get

$$
\begin{aligned}
& -\sum_{j=1}^{n} \theta_{j} \varepsilon\left(\sum_{i=1}^{N_{j}} \frac{\left(e_{j, i+1}-e_{j, i}\right)^{2}}{h_{i+1 / 2}^{e_{j}}}+\frac{\left(e_{j, 1}-e_{j, 0}\right)^{2}}{h_{1 / 2}^{e_{j}}}\right)-\sum_{\substack{\sigma \in \mathcal{E}_{i n t} \\
\sigma=\sigma_{K / L}}} \frac{m(\sigma)}{d_{\sigma}}\left(e_{L}-e_{K}\right)^{2}-\sum_{j=1}^{n} \sum_{\substack{\sigma \in \mathcal{E}_{K} \\
\sigma \subset \gamma_{j}^{\prime}}} \frac{m(\sigma)}{d_{\sigma}}\left(e_{j, 0}-e_{K}\right)^{2} \\
& =\sum_{j=1}^{n} \theta_{j} \varepsilon \sum_{i=1}^{N_{j}} R_{j, i+1 / 2}\left(e_{j, i}-e_{j, i+1}\right)-\sum_{j=1}^{n} \theta_{j} \varepsilon R_{j, 1 / 2}^{\nabla}\left(e_{j, 1}-e_{j, 0}\right)+\sum_{\substack{\sigma \in \mathcal{E}_{i n t} \\
\sigma=\sigma_{K / L}}} m(\sigma) R_{K, \sigma}\left(e_{K}-e_{L}\right) \\
& +\sum_{j=1}^{n} \sum_{\substack{\sigma \in \mathcal{E}_{K} \\
\sigma \subset \gamma_{j}^{\prime}}} m(\sigma) R_{K, \sigma}^{\nabla}\left(e_{K}-e_{j, 0}\right) .
\end{aligned}
$$

We recognize $-\left\|e_{\mathcal{T}}\right\|_{1, \mathcal{T}}^{2}$ in the left member of (4.21). We then apply Cauchy-Schwarz inequality. This gives

$$
\begin{aligned}
\left\|e_{\mathcal{T}}\right\|_{1, \mathcal{T}} \leq & \left(\sum_{j=1}^{n} \theta_{j} \varepsilon \sum_{i=1}^{N_{j}} R_{j, i+1 / 2}^{2} h_{i+1 / 2}^{e_{j}}+\sum_{j=1}^{n} \theta_{j} \varepsilon\left(R_{j, 1 / 2}^{\nabla}\right)^{2} h_{1 / 2}^{e_{j}}+\sum_{\sigma \in \mathcal{E}_{i n t}, \sigma=\sigma_{K / L}} m(\sigma) d_{\sigma} R_{K, \sigma}^{2}\right. \\
& \left.+\sum_{j=1}^{n} \sum_{\sigma \in \mathcal{E}_{K}, \sigma \subset \gamma_{j}^{\prime}} m(\sigma) d_{\sigma}\left(R_{K, \sigma}^{\nabla}\right)^{2}\right)^{1 / 2} \\
\leq & h O\left(\left\|v^{\prime \prime}\right\|_{\infty}+\left\|\nabla^{2} u\right\|_{\infty}\right)\left(\sum_{j=1}^{n} \theta_{j} \varepsilon \sum_{i=0}^{N_{j}} h_{i+1 / 2}^{e_{j}}+\sum_{\sigma \in \mathcal{E}_{i n t}} m(\sigma) d_{\sigma}+\sum_{j=1}^{n} \sum_{\sigma \subset \gamma_{j}^{\prime}} m(\sigma) d_{\sigma}\right)^{1 / 2} \\
\leq & h O\left(\left\|v^{\prime \prime}\right\|_{\infty}+\left\|\nabla^{2} u\right\|_{\infty}\right)\left(\sum_{j=1}^{n} \theta_{j} \varepsilon\left(l_{j}-\delta\right)+2 m\left(\Omega_{\varepsilon}^{\prime}\right)\right)^{1 / 2} .
\end{aligned}
$$

Since the solution is regular, the second derivatives of the solution are bounded. We conclude that there is a constant $c_{1}$ depending only on $u^{d}$ and $D_{\varepsilon}$ such that

$$
\left\|e_{\mathcal{T}}\right\|_{1, \mathcal{T}} \leq c_{1} h
$$

With the Poincaré inequality (Lem. 3.3), this yields

$$
\left\|e_{\mathcal{T}}\right\|_{2, \mathcal{T}} \leq \sqrt{c} c_{1} h .
$$

Let $\hat{u}_{\mathcal{T}}$ be a function defined for a.e. $(x, y) \in \Omega_{\varepsilon}$ by

$$
\hat{u}_{\mathcal{T}}(x, y)=\left\{\begin{array}{l}
u\left(x_{K}\right),(x, y) \in K, K \in \mathcal{T} \\
v_{j}\left(x_{i}^{e_{j}}\right),(x, y) \in B_{j}^{\varepsilon}, x^{e_{j}} \in\left(x_{i-1 / 2}^{e_{j}}, x_{i+1 / 2}^{e_{j}}\right), i=1, \ldots, N_{j}, j=1, \ldots, n .
\end{array}\right.
$$

We have

$$
\left\|u^{d}-u_{\mathcal{T}}^{d}\right\|_{L^{2}\left(\Omega_{\varepsilon}\right)} \leq\left\|u^{d}-\hat{u}_{\mathcal{T}}\right\|_{L^{2}\left(\Omega_{\varepsilon}\right)}+\left\|e_{\mathcal{T}}\right\|_{2, \mathcal{T}}
$$

and

$$
\left\|u^{d}-\hat{u}_{\mathcal{T}}\right\|_{L^{2}\left(\Omega_{\varepsilon}\right)} \leq h O\left(\left\|v^{\prime}\right\|_{\infty}+\|\nabla u\|_{\infty}\right)\left(\sum_{j=1}^{n} \theta_{j} \varepsilon\left(l_{j}-\delta\right)+m\left(\Omega_{\varepsilon}^{\prime}\right)\right)^{1 / 2} .
$$


Using (4.25), since the solution is regular, the estimates (4.23) and (4.26) yield (4.2). This ends the proof of Theorem 4.1.

Remark 4.3. It is also possible to prove the estimates of Theorem 4.1 under the weaker assumption $u \in$ $H^{2}\left(\Omega_{\varepsilon}^{\prime}\right), v_{j} \in H^{2}\left(\left(\delta, l_{j}\right)\right), j=1, \ldots, n$. Taylor expansions with integral errors should be used to bound the consistency errors. The bounds (4.8) and (4.10) (resp. (4.22)) should involve the $L^{2}$-norm of the second derivatives in some part of the corresponding control volumes (resp. in $\Omega_{\varepsilon}^{\prime}$ and $\left(\delta, l_{j}\right)$ ), and (4.1) should still be true (see [19]).

Now, Theorem 4.1 yields the following estimate about the solution of (1.3).

Theorem 4.4. Let $u_{\mathcal{T}}^{d}$ be the finite volume approximation of (1.1) defined by (2.4). For any $J>0$, there is $M$ independent of $\varepsilon$, such that if $\delta=M \varepsilon|\ln \varepsilon|$, if the solution of (1.3) is assumed to be regular, then we have

$$
\left\|u_{\varepsilon}-u_{\mathcal{T}}^{d}\right\|_{L^{2}\left(\Omega_{\varepsilon}\right)}=O\left(\frac{h}{\sqrt{\varepsilon}}\right)+O\left(\varepsilon^{J}\right) .
$$

Proof. Using Lemma 1.2 we deduce in this case from (4.22) that

$$
\left\|e_{\mathcal{T}}\right\|_{1, \mathcal{T}}=h O\left(\frac{1}{\varepsilon}\right) O(\sqrt{\varepsilon})=O\left(\frac{h}{\sqrt{\varepsilon}}\right) .
$$

With Lemma 3.3, this yields

$$
\left\|e_{\mathcal{T}}\right\|_{2, \mathcal{T}}=O\left(\frac{h}{\sqrt{\varepsilon}}\right) .
$$

We deduce from (4.26) that

$$
\left\|u^{d}-\hat{u}_{\mathcal{T}}\right\|_{L^{2}\left(\Omega_{\varepsilon}\right)}=h O(1) O(\sqrt{\varepsilon})
$$

so this term is negligible compared with (4.27) if $\varepsilon$ is small, and (4.25) yields

$$
\left\|u^{d}-u_{\mathcal{T}}^{d}\right\|_{L^{2}\left(\Omega_{\varepsilon}\right)}=O\left(\frac{h}{\sqrt{\varepsilon}}\right) .
$$

We end the proof by applying Lemma 1.1.

Remark 4.5. If $\varepsilon$ is fixed and small so that $O\left(\varepsilon^{J}\right)$ is negligible, then $\left\|u_{\varepsilon}-u_{\mathcal{T}}^{d}\right\|_{L^{2}\left(\Omega_{\varepsilon}\right)}=O(h)$, that improves the convergence order $O(\sqrt{h})$ in terms of the size of the mesh that we get in [36] for (1.3) (see Sect. 1.5).

Moreover, it is reasonable to expect that the mesh of $\Omega_{\varepsilon}^{\prime}$ is not excessively coarse near the interfaces. So the assumption $h<\theta_{\min } \varepsilon, \theta_{\min }=\min \left\{\theta_{j}, j=1, \ldots, n\right\}$ is not restrictive, especially since $h$ is intended to be small. That is why it is worth highlighting that:

Lemma 4.6. Under the assumptions of Theorem 4.4, if there is a constant $c$ such that $h<c \varepsilon$ then

$$
\left\|u_{\varepsilon}-u_{\mathcal{T}}^{d}\right\|_{L^{2}\left(\Omega_{\varepsilon}\right)}=O(\sqrt{\varepsilon})
$$

So the convergence order in terms of $\varepsilon$ is the same as the one we obtained in [36] using another kind of proof. If we look at the numerical experiments in [36], the convergence order in terms of $\varepsilon$ seems to be optimal.

Remark 4.7. It is possible to consider that $u^{d}$ is defined on a domain $\Omega_{\varepsilon}$ with regular boundary and that $u_{\mathcal{T}}^{d}$ is defined on a polygonal domain $\Omega_{\varepsilon, p o l y} \subset \Omega_{\varepsilon}$. This would increase the number of possible regular solutions of (1.1). In this case, the results of Theorem 4.1, Theorem 4.4, Remark 4.5 and Lemma 4.6 are still valid in $L^{2}\left(\Omega_{\varepsilon, p o l y}\right)$.

The hybrid scheme has been used to solve the Poisson equation in [36]. The numerical experiments have shown that the numerical convergence order of the hybrid scheme seems to be greater than one. So the theoretical convergence order $O(h)$, in terms of the size of the mesh, that we obtained in the present paper, seems not yet optimal. However, we also observe a difference between the numerical and the theoretical convergence order for other schemes for instance the TPFA scheme (defined in [19], see [15]). 


\section{Conclusion}

In this paper, we study a finite volume scheme to solve a linear model problem on a geometrical multi-scale 1D-2D domain (one node and $n$ outgoing branches). This could contribute to the simulation of problems set in rod structures, such as arterial trees for example. Indeed this study can be generalized to solve more realistic problems (the heat equation will be addressed in a forthcoming paper) which are possibly set in a 3D rodstructure. We explain what are the advantages to work on a dimensionally-heterogeneous domain rather than keep a 2D domain and consider non-matching grids. We define a specific $H^{1}$ discrete norm for the functions defined on such a domain, which involves the convex combinations of the values of the functions on both sides of the interfaces between the 1D part and the 2D part. We establish a Poincaré inequality that yields a $L^{2}$ error estimate (4.2). If the thickness of the branches, that is proportional to $\varepsilon$, is fixed, then this estimate can be read in terms of the size of the mesh $h$ (order of convergence 1). If not, this estimate is rewritten (4.29) and it can be read in terms of $\varepsilon$ (order of convergence 1/2). Indeed, the convergence according to the thickness of the branches may also be considered when applying the Method of Asymptotic Partial Domain Decomposition, since then the thickness of the branches is intended to tend to zero. To the best of our knowledge, we prove here the first error estimate using a finite volume scheme in a geometrical multi-scale domain.

Acknowledgements. The author would like to thank the reviewers for their careful and constructive assessment of the manuscript.

\section{REFERENCES}

[1] Y. Achdou, C. Japhet, Y. Maday and F. Nataf, A new cement to glue non-conforming grids with Robin interface conditions: the finite volume case. Numer. Math. 92 (2002) 593-620.

[2] A. Ambroso, C. Chalons, F. Coquel, E. Godlewski, F. Lagoutière, P.A. Raviart and N. Seguin, Relaxation methods and coupling procedures. Int. J. Numer. Methods Fluids 56 (2008) 1123-1129.

[3] B. Andreianov, F. Boyer and F. Hubert, Discrete duality finite volume schemes for Leray-Lions-type elliptic problems on general 2D meshes. Numer. Method Partial Differ. Eq. 23 (2007) 145-195.

[4] B. Andreianov, M. Bendahmane and R. Ruiz Baier, Analysis of a finite volume method for a cross-diffusion model in population dynamics. Math. Meth. Appl. Sci. 21 (2011) 307-344.

[5] M. Bessemoulin-Chatard, C. Chainais-Hillairet and F. Filbet, On discrete functional inequalities for some finite volume schemes. To appear in IMA J. Numer. Anal. (2014).

[6] P.J. Blanco, R.A. Feijóo and S.A. Urquiza, A unified variational approach for coupling 3D-1D models and its blood flow applications. Comput. Methods Appl. Mech. Eng. 196 (2007) 4391-4410.

[7] P.J. Blanco, J.S. Leiva, R.A. Feijóo and G.S. Buscaglia, Black-box decomposition approach for computational hemodynamics: One-dimensional models. Comput. Methods Appl. Mech. Eng. 200 (2011) 1389-1405.

[8] P.J. Blanco, M.R. Pivello, S.A. Urquiza and R.A. Feijóo, On the potentialities of 3D-1D coupled models in hemodynamics simulations. J. Biomech. 42 (2009) 919-930.

[9] P.J. Blanco, S.A. Urquiza and R.A. Feijóo, Assessing the influence of heart rate in local hemodynamics through coupled 3D-1D-0D models. Int. J. Numer. Methods Biomed. Eng. 26 (2010) 890-903.

[10] B. Boutin, C. Chalons and P.A. Raviart, Existence result for the coupling problem of two scalar conservation laws with Riemann initial data. Math. Models Methods Appl. Sci. 20 (2010) 1859-1898.

[11] R. Cautrés, R. Herbin and F. Hubert, The Lions domain decomposition algorithm on non matching cell-centred finite volume meshes. IMA J. Numer. Anal. 24 (2004) 465-490.

[12] C. Chainais-Hillairet and J. Droniou, Finite volume schemes for non-coercive elliptic problems with Neumann boundary conditions. IMA J. Numer. Anal. 31 (2011) 61-85.

[13] Y. Coudière, J.-P. Vila and P. Villedieu, Convergence rate of a finite volume scheme for a two-dimensional convection-diffusion problem. ESAIM: M2AN 33 (1999) 493-516.

[14] Y. Coudière, T. Gallouët and R. Herbin, Discrete Sobolev Inequalities and $L^{p}$ error estimates for finite volume solutions of convection diffusion equations. ESAIM: M2AN 35 (2001) 767-778.

[15] K. Domelevo and P. Omnes, A finite volume method for the Laplace equation on almost arbitrary two-dimensional grids. ESAIM: M2AN 39 (2005) 1203-1249.

[16] M. Deininger, J. Jung, R. Skoda, P. Helluy and C.-D. Munz, Evaluation of interface models for 3D-1D coupling of compressible Euler methods for the application on cavitating flows. CEMRACS'11: Multiscale coupling of complex models in scientific computing. ESAIM Proceedings. EDP Sciences Les Ulis 38 (2012) 298-318.

[17] J. Droniou, T. Gallouët and R. Herbin, A finite volume scheme for a noncoercive elliptic equation with measure data. SIAM J. Numer. Anal. 41 (2003) 1997-2031. 
[18] R. Eymard, T. Gallouët and R. Herbin, Discretization of heterogeneous and anisotropic diffusion problems on general nonconforming meshes SUSHI: a scheme using stabilization and hybrid interfaces. IMA J. Numer. Anal. 30 (2010) 1009-1043.

[19] R. Eymard, T. Gallouët and R. Herbin, Finite Volume Methods. Handb. Numer. Anal. Edited by P.G. Ciarlet and J.L. Lions (2000).

[20] F. Filbet, A finite volume scheme for the Patlak-Keller-Segel chemotaxis model. Numer. Math. 104 (2006) $457-488$.

[21] F. Fontvieille, G.P. Panasenko and J. Pousin, FEM implementation for the asymptotic partial decomposition. Appl. Anal. Int. J. 86 (2007) 519-536.

[22] L. Formaggia, F. Nobile, A. Quarteroni and A. Veneziani, Multiscale modelling of the circulatory system: a preliminary analysis. Comput. Visual. Sci. 2 (1999) 75-83.

[23] L. Formaggia, J.F. Gerbeau, F. Nobile and A. Quarteroni, On the coupling of 3D and 1D Navier-Stokes equations for flow problems in compliant vessels. Comput. Methods Appl. Mech. Eng. 191 (2001) 561-582.

[24] L. Formaggia, A. Quarteroni and A. Veneziani, Cardiovascular Mathematics, Series: Model. Simul. Appl., vol. 1. Springer (2009).

[25] T. Gallouët, R. Herbin and M.H. Vignal, Error estimates on the approximate finite volume solution of convection diffusion equations with general boundary conditions. SIAM J. Numer. Anal. 37 (2000) 1935-1972.

[26] A. Glitzky and J.A. Griepentrog, Discrete Sobolev-Poincaré Inequalities for Voronoi Finite Volume Approximations. SIAM J. Numer. Anal. 48 (2010) 372-391.

[27] P. Grisvard, Elliptic Problems in Non Smooth Domains. Pitman (1985).

[28] J.M. Hérard and O. Hurisse, Coupling two and one-dimensional unsteady Euler equations through a thin interface. Comput. Fluids 36 (2007) 651-666.

[29] R. Herbin, An error estimate for a finite volume scheme for a diffusion-convection problem on a triangular mesh. Numer. Method Partial Differ. Eq. 11 (1995) 165-173.

[30] J. Heywood, R. Rannacher and S. Turek, Artificial boundaries and flux and pressure conditions for the incompressible NavierStokes equations. Int. J. Num. Meth. Fl. 22 (1996) 325-352.

[31] A.H. Le and P. Omnes, Discrete Poincaré inequalities for arbitrary meshes in the discrete duality finite volume context. Electronic Trans. Numer. Anal. 40 (2013) 94-119.

[32] J.S. Leiva, P.J. Blanco and G.S. Buscaglia, Iterative strong coupling of dimensionally heterogeneous models. Int. J. Numer. Methods Eng. 81 (2010) 1558-1580.

[33] J.S. Leiva, P.J. Blanco and G.S. Buscaglia, Partitioned analysis for dimensionally-heterogeneous hydraulic networks. SIAM Multiscale Model. Simul. 9 (2011) 872-903.

[34] A.C.I. Malossi, P.J. Blanco, P. Crosetto, S. Deparis and A. Quarteroni, Implicit coupling of one-dimensional and threedimensional blood flow models with compliant vessels. Multiscale Model. Simul. 11 (2013) 474-506.

[35] G.P. Panasenko, Method of asymptotic partial decomposition of domain. Math. Models Methods Appl. Sci. 8 (1998) 139-156.

[36] G.P. Panasenko and M.-C. Viallon, Error estimate in a finite volume approximation of the partial asymptotic domain decomposition. Math. Meth. Appl. Sci. 36 (2013) 1892-1917.

[37] G.P. Panasenko and M.-C. Viallon, The finite volume implementation of the partial asymptotic domain decomposition. Appl. Anal. Int. J. 87 (2008) 1397-1424.

[38] T. Passerini, M. de Luca, L. Formaggia and A. Quarteroni, A 3D/1D geometrical multiscale model of cerebral vasculature. J. Eng. Math. 64 (2009) 319-330.

[39] A. Quarteroni and L. Formaggia, Mathematical Modelling and Numerical Simulation of the Cardiovascular System. Modelling of Living Systems. Edited by N. Ayache. Handb. Numer. Anal. Series (2002).

[40] L. Saas, I. Faille, F. Nataf and F. Willien, Finite volume methods for domain decomposition on non matching grids with arbitrary interface conditions. SIAM J. Numer. Anal. 43 (2005) 860-890.

[41] S.A. Urquiza, P.J. Blanco, M.J. Vénere and R.A. Feijóo, Multidimensional modelling for the carotid artery blood flow. Comput. Methods Appl. Mech. Eng. 195 (2006) 4002-4017.

[42] M.-C. Viallon, Error estimate for a 1D-2D finite volume scheme. Comparison with a standard scheme on a 2D non-admissible mesh. C. R. Acad. Sci. Paris, Ser. I 351 (2013) 47-51.

[43] M. Vohralik, On the discrete Poincaré-Friedrichs inequalities for nonconforming approximations of the sobolev space $H^{1}$. Numer. Funct. Anal. Optim. 26 (2005) 925-952.

[44] M. Vohralik, Numerical methods for nonlinear elliptic and parabolic equations. Application to flow problems in porous and fractured media. Ph.D. thesis, Université de Paris-Sud and Czech Technical University in Prague.

[45] S.M. Watanabe, P.J. Blanco and R.A. Feijóo, Mathematical model of blood flow in an anatomically detailed arterial network of the arm. ESAIM: M2AN $\mathbf{4 7}$ (2013) 961-985. 